\title{
Second spectrum of chromium (Cr II), Part III: Radiative lifetimes and transition probabilities from highly excited $3 \mathrm{~d}^{4} 5$ s levels
}

\author{
Safa Bouazza ${ }^{\mathrm{a}, *}$, Patrick Palmeri ${ }^{\mathrm{b}}$, Pascal Quinet ${ }^{\mathrm{b}, \mathrm{c}}$ \\ a Département de Physique, Université de Reims-Champagne, UFR SEN, BP 1039 F-51687 Reims Cedex2, France \\ b Physique Atomique et Astrophysique, Université de Mons-UMONS, B-7000 Mons, Belgium \\ c IPNAS, Université de Liège, B-4000 Liège, Belgium
}

A R T I C L E I N F O

\section{Article history:}

Received 9 May 2017

Received in revised form 15 May 2017

Accepted 15 May 2017

Available online 17 June 2017

\section{Keywords:}

Cr II spectrum

$3 d^{4} 5$ s configuration

Oscillator strengths

Lifetime

Transition probabilities

Branching fractions

Transition radial integrals

Semi-empirical calculations

\begin{abstract}
A B S T R A C T
In this study we have extended our previous investigations of $\mathrm{Cr}$ II spectrum to levels of the $3 \mathrm{~d}^{4} 5 \mathrm{~s}$ configuration. Once again an overall good agreement is observed for these levels between experimental oscillator strength values and our calculated data using two different approaches, namely the oscillator strength parameterization and the HFR+CPOL methods. Recurring to the latter model we have computed radiative lifetime values for $203 \mathrm{~d}^{4} 5$ s levels, confirming the well founded basis of recent experimental data, given in literature. From the use of the former method and with the help of a least squares fitting procedure to available experimental gf-values we have taken advantage of extracting for the first time the transition radial integral value for $3 \mathrm{~d}^{4} 4 \mathrm{p}-3 \mathrm{~d}^{4} 5 \mathrm{~s},\left\langle 4 p\left|r^{1}\right| 5 \mathrm{~s}\right\rangle=1.962 \pm 0.005$, which is confirmed favorably by $a b$ initio results, obtained by the HFR method scaled by a factor 0.92 or directly by the HFR+CPOL approach. Finally a long list of semi-empirical oscillator strength, transition probability and branching fraction values is generated for around $500 \mathrm{Cr}$ II transitions depopulating the $3 \mathrm{~d}^{4} 5$ s levels. Our new gf data are compared successfully with those given by Kurucz.
\end{abstract}

(c) 2017 Elsevier Inc. All rights reserved.

\footnotetext{
* Corresponding author.

E-mail address: safa.bouazza@univ-reims.fr (S. Bouazza).
} 
Contents

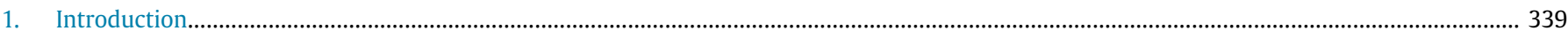

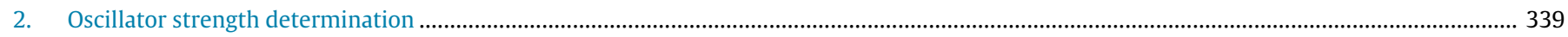

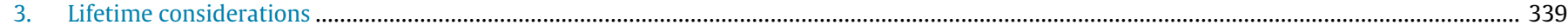

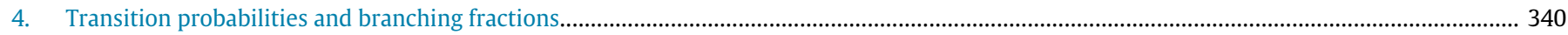

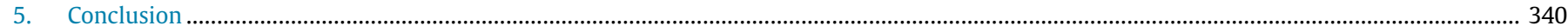

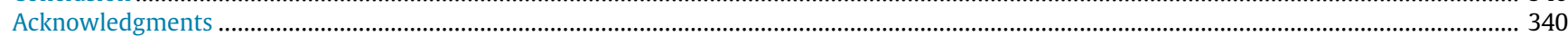

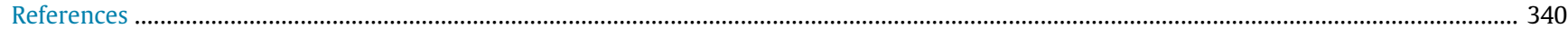

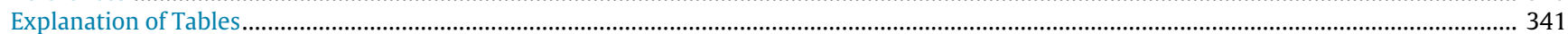

Table 1. Eigenvalue components of even-parity $\mathrm{Cr}$ II levels of interest in this study, more detailed than in our previous paper [1]. ......... 341

Table 2. Comparison between experimental and calculated oscillator strength values ............................................................................... 341

Table 3. Comparison between HFR+CPOL radiative lifetimes (in ns) and available experimental data for low-lying $3 \mathrm{~d}^{4} 5 \mathrm{~s}$ energy levels of $\mathrm{Cr}$ II.

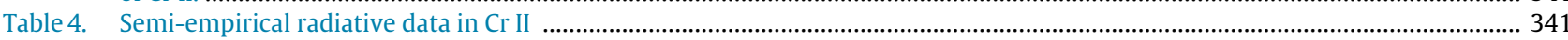

\section{Introduction}

For many years we have performed atomic structure calculations in order to provide astrophysicists with new or improved radiative data in different ions. Such data play a key role in the investigation and the interpretation of the observed spectra produced by any type of celestial objects. This spectroscopic analysis, with the help of reliable radiative parameters, can be used to derive many properties of distant stars and galaxies, such as their chemical composition, temperature, density, mass, distance and luminosity, for example. Previously we have successfully studied the electronic structure of many different complex atomic systems. The present work is devoted to the calculation of oscillator strengths and transition probabilities in singly ionized chromium, paying a particular attention to transitions depopulating highly excited $3 \mathrm{~d}^{4} 5 \mathrm{~s}$ levels. This paper is the third part of a detailed and systematic analysis of the Cr II spectrum. As a reminder, in the first part [1] we have studied the fine structure in order to obtain level eigenvector to transform into actual intermediate coupling the transition matrix beforehand obtained in pure LS coupling with help of Racah algebra while, in the second part [2], our efforts were mainly focused on radiative transitions depopulating lowlying $3 \mathrm{~d}^{4} 4 \mathrm{p}$ levels. For $\mathrm{Cr}$ II the most recent compilation of energy levels is due to Sugar \& Corliss [3], based on the analysis of Kiess [4] who succeeded in classifying 1843 lines linking 138 even-parity levels of $3 d^{5}, 3 d^{4} 4 s$ and $3 d^{3} 4 s^{2}$ configurations to $1393 d^{4} 4 p$ oddparity levels. Johansson [5] extended the Kiess study, particularly in near-infrared region and analyzed 450 additional levels. Sansonetti et al. [6-8] reported in turn new observations of Cr II some years later, in the near-ultraviolet region 1140-3400 $\AA$, and also up to the infrared region: 2850-37900 $\AA$, using $10.7 \mathrm{~m}$ normal incidence vacuum spectrograph and FT700 vacuum ultraviolet Fourier transform spectrometer. In our recent study of the fine structure in $\mathrm{Cr}$ II [1], we notably revised the assignments of some levels wrongly classified in earlier lists of energy levels. We also shifted the positions of some quartets like $3 d^{4} 5 d{ }^{4} F_{J}$ for instance and we predicted the positions of still missing levels. Nevertheless we have to point out that $3 \mathrm{~d}^{4} 5 \mathrm{~s}$ levels were correctly assigned in previous studies and we have not changed any label of these levels. To compute oscillator strength values we used two different approaches, namely the HFR+CPOL model $[9,10]$ and oscillator strength parameterization (OSP) method $[11,12]$. For the latter we need experimental data since transition radial integrals are treated as free parameters in the least squares fit to experimental gf values. Here we take advantage of the experimental work of a Swedish team from Lund University [13] which reported lifetime measurements of five $3 \mathrm{~d}^{4} 5 \mathrm{~s}{ }^{6} \mathrm{D}$ levels at energy around $83000 \mathrm{~cm}^{-1}$ and $\log$ gf values for 38 transitions from these levels. The lifetimes were obtained using time-resolved laser-induced fluorescence and gf-values were deduced by combining the experimental lifetimes with relative intensities, i.e. branching fractions.

\section{Oscillator strength determination}

The reader is invited to read one of our previous papers, for instance $[2,14,15]$, in which the formulas and the approaches we have used to achieve our calculations are presented in detail. Usually before performing OSP calculations, we first have to select the strongest lines, not blended, and preferably those corresponding to transitions between levels with a limited number of leading components. In the present work we have not selected beforehand some lines but we have considered the complete set of experimental gf-values for the thirty eight transitions involving $3 d^{4} 5$ s levels listed in [13]. The compositions of the eigenvectors obtained in the present work for these levels are displayed in Table 1. For odd-parity levels the compositions were already given in our previous paper [1]. When comparing our fitted data to the experimental oscillator strengths, a very satisfactory agreement is observed on the whole except for the lines whose wavelengths are: $\lambda_{\text {air }}=2787.914,2899.195,2945.277,2967.410,3013.455$ and $3194.200 \AA$ A. Moreover, our OSP results are in close agreement with those obtained using our HFR+CPOL model, as described in [2], and with those reported by Kurucz [16]. Such comparisons can be found in Table 2. So we suggest that the experimental gf values of lines whose wavelengths are here above mentioned should be re-examined. From our semi-empirical fitting process, we have extracted the main transition radial integral value with a very good accuracy: $\left\langle 4 p\left|r^{1}\right| 5 s\right\rangle=1.962 \pm 0.005$, which corresponds to the HFR+CPOL value (1.969) or the HFR value computed by classical Cowan code [17], i.e. 2.139 , scaled by a factor 0.92 . This confirms the trends we already highlighted in [15] for several singly ionized ions.

\section{Lifetime considerations}

Some years ago Engman et al. [18] and Pinnington et al. [19] have used the beam-foil technique to measure radiative lifetimes of low-lying levels of $\mathrm{Cr}$ II $3 \mathrm{~d}^{4}\left({ }^{5} \mathrm{D}\right) 4 \mathrm{p}$ configuration. Later Schade et al. [20], Pinnington et al. [21], Bergeson and Lawler [22] and Nilsson et al. [23] have extended these experimental data using time-resolved laser-induced fluorescence method (TR-LIF) on ions produced in a fast ion beam in a hollow cathode from laserprocured plasma but unfortunately for levels belonging to the same $3 \mathrm{~d}^{4} 4 \mathrm{p}$ configuration. It is only in 2014 that Engström et al. [13] have reported for the first time lifetime measurements of five levels in the $3 \mathrm{~d}^{4} 5 \mathrm{~s}$ configuration, all of them belonging to the same ${ }^{6} \mathrm{D}$ multiplet located around $83000 \mathrm{~cm}^{-1}$. These data are gathered in Table 3 and compared to HFR+CPOL radiative lifetimes. We could expect some discrepancies between our calculated data and those given in [13] since we have observed for few transitions some divergences between our calculated gf and those given in [13] 
which were extracted from a combination of lifetimes and branching fractions. As seen from Table 3 a good agreement is observed, the mean ratio $\tau_{\text {exp }} / \tau_{\text {HFR }+ \text { CPOL }}$ being found equal to 0.91 . We can then conclude that the discrepancies mentioned in Section II for some gf-values are rather due to branching fractions; Engström et al. have corrected the effects of missing branches on their measurements using the unpublished theoretical values computed by Raassen \& Uylings reported in their paper [13].

\section{Transition probabilities and branching fractions}

Finally, in Table 4, we give the oscillator strengths, transition probabilities and branching fractions computed in the present work using the HFR+CPOL method for about $500 \mathrm{Cr}$ II spectral lines involving available experimental energy levels with calculated $\log$ gf values greater than -4 . This table covers a wide range of wavelengths, from 1750 to $8700 \AA$ for $3 d^{4} 4 p-3 d^{4} 5$ s transitions. We have taken this opportunity to compare our semi-empirical data with those given by Kurucz [16]. We note a good agreement between these different sets of results. To derive branching fraction values we have taken experimental lifetime values for levels whose energies are: $82692,82763,82881,83041$ and $83240 \mathrm{~cm}^{-1}$. Unfortunately for the other $3 \mathrm{~d}^{4} 5 \mathrm{~s}$ levels no experimental lifetime values are available in literature and then we have replaced these missing values by those computed by means of HFR+CPOL model (see Table 1).

\section{Conclusion}

After studying radiative parameters of low-lying Cr II $3 d^{4} 4 p$ levels [2], we have investigated those of higher excited $3 d^{4} 5$ s levels with the same success. Using the oscillator strength parameterization method, we have fitted the computed gf-values to available experimental data, confirming in the whole their well founded basis. We have taken this opportunity to extract for the first time the $\left\langle 4 p\left|r^{1}\right| 5 s\right\rangle$ transition radial integral value with a very good accuracy. Using the HFR+CPOL approach, we have calculated the radiative lifetimes of $3 \mathrm{~d}^{4} 5 \mathrm{~s}$ levels and compared them to the few available experimental ones. In addition, a list of oscillator strength, transition probability and branching fraction values of about 500 transitions involving $3 \mathrm{~d}^{4} 5$ s levels was generated. These new computed radiative decay rates agree very well with those obtained by Kurucz [16], so supplying an even more solid basis to all the spectroscopic parameters determined until now in singly ionized chromium. These very encouraging results incite us to extend the present work to $\mathrm{Cr}$ II transitions involving $3 \mathrm{~d}^{4} 4 \mathrm{~d}, 3 \mathrm{~d}^{4} n \mathrm{f}$ $(n=4,5)$ and highly excited $3 \mathrm{~d}^{4} 4$ p levels.

\section{Acknowledgments}

PQ and PP are respectively Research Director and Research Associate of the Belgian Fund for Scientific Research F.R.S.-FNRS, Financial support from this organization is deeply acknowledged.

\section{References}

[1] S. Bouazza, P. Quinet, P. Palmeri, ADNDT, http://dx.doi.org/10.1016/j.adt.2017. 05.003.

[2] S. Bouazza, P. Quinet, P. Palmeri, ADNDT, http://dx.doi.org/10.1016/j.adt.2017. 05.008 .

[3] J. Sugar, C. Corliss, J. Phys. Chem. Ref. Data Suppl. 14 (1985) 264.

[4] C.C. Kiess, J. Res. Natl. Bur. Stand. 60 (1958) 375 RP2856.

[5] S. Johansson, T. Zethson, H. Hartman, et al., Astron. Astrophys. 361 (2000) 977.

[6] C.J. Sansonetti, F. Kerber, J. Reader, M.R. Rosa, Astrophys. J. Suppl. Ser. 153 (2004) 555

[7] C.J. Sansonetti, G. Nave, J. Reader, F. Kerber, Astrophys. J. Suppl. Ser. 202 (2012) 15.

[8] C.J. Sansonetti, G. Nave, Astrophys. J. Suppl. Ser. 213 (2014) 1.

[9] P. Quinet, P. Palmeri, E. Biémont, M.M. McCurdy, G. Rieger, E.H. Pinnington, M.E. Wickliffe, J.E. Lawler, Mon. Not. R. Astron. Soc. 307 (1999) 934.

[10] P. Quinet, P. Palmeri, E. Biémont, Z.S. Li, Z.G. Zhang, S. Svanberg, J. Alloys Compd. 344 (2002) 255

[11] J. Ruczkowski, M. Elantkowska, J. Dembczynski, J. Quant. Spectrosc. Radiat. Transfer 145 (2014) 20.

[12] S. Bouazza, J. Ruczkowski, M. Elantkowska, J. Dembczynski, J. Quant. Spectrosc Radiat. Transfer 166 (2015) 55

[13] L. Engström, H. Lundberg, H. Nilsson, H. Hartman, E. Bäckström, Astron. Astrophys. 570 (2014) A34

[14] S. Bouazza, J. Quant. Spectrosc. Radiat. Transfer 187 (2017) 280

[15] S. Bouazza, P. Quinet, P. Palmeri, J. Quant. Spectrosc. Radiat. Transfer. 199 (2017) 66

[16] R.L. Kurucz, Atomic spectral line data from CD-ROM Nr. 23, 1995. http://www cfa.harvard.edu/amp/ampdata/kurucz23/sekur.html.

[17] R.D. Cowan, The Theory of Atomic Structure Spectra, University of California Press, Berkeley, CA, 1981.

[18] B. Engman, A. Gaupp, L.J. Curtis, I. Martinson, Phys. Scr. 12 (1975) 220.

[19] E.H. Pinnington, H.O. Lutz, G.W. Carriaveao, Nucl. Instrum. Methods 110 (1973) 55.

[20] W. Schade, B. Mundt, V. Helbig, Phys. Rev. A 42 (1990) 3.

[21] E.H. Pinnington, Q. Ji, B. Gou, et al., Can. J. Phys. 71 (1993) 470.

[22] S.D. Bergeson, J.E. Lawler, Astrophys. J. 408 (1993) 382.

[23] H. Nilsson, G. Lyung, H. Lundberg, K.E. Nielsen, Astron. Astrophys. 445 (2006) 1165 


\section{Explanation of Tables}

Table 1. Eigenvalue components of even-parity $\mathrm{Cr}$ II levels of interest in this study, more detailed than in our previous paper [1].

Level: $\quad$ Configuration to which belongs the even-parity level involved in the studied transitions and the designation of this level in LS

$\begin{array}{ll}\text { Coupling. } & \text { Total angular momentum. }\end{array}$

Energy: $\quad$ Experimental energy level $\left(\mathrm{cm}^{-1}\right)$.

$3 d^{5}$ : Contribution in LS coupling of $3 d^{5}$ configuration (in \%) to the total composition of the level.

$3 \mathrm{~d}^{4} 4 \mathrm{~s}$ : $\quad$ Contribution in LS coupling of $3 \mathrm{~d}^{4} 4 \mathrm{~s}$ configuration (in \%) to the total composition of the level.

$3 \mathrm{~d}^{4} 4 \mathrm{~d}$ : $\quad$ Contribution in LS coupling of $3 \mathrm{~d}^{4} 4 \mathrm{~d}$ configuration (in \%) to the total composition of the level.

$3 d^{4} 5 s$ : Contribution in LS coupling of $3 d^{4} 5$ s configuration (in \%) to the total composition of the level.

$3 \mathrm{~d}^{4} 6 \mathrm{~s}$ : $\quad$ Contribution in LS coupling of $3 \mathrm{~d}^{4} 6 \mathrm{~s}$ configuration (in \%) to the total composition of the level.

$3 d^{4} 5 d$ : Contribution in LS coupling of $3 d^{4} 5 d$ configuration (in \%) to the total composition of the level.

$3 \mathrm{~d}^{4} 6 \mathrm{~d}$ : $\quad$ Contribution in LS coupling of $3 \mathrm{~d}^{4} 6 \mathrm{~d}$ configuration (in \%) to the total composition of the level.

Others: $\quad$ Total contribution in LS coupling of other configurations (in \%) to the total composition of the level.

Table 2. Comparison between experimental and calculated oscillator strength values

$\lambda_{\text {air }}$ : $\quad$ Wavelength in the air of the spectral line $(\AA ̊)$ deduced from observed energy levels taken from [13].

Upper level: $\quad$ Depopulated upper level [13] $\left(\mathrm{cm}^{-1}\right)$ in emission transition.

$J_{U}$ : Total angular momentum of the upper level.

Lower level: $\quad$ Populated lower level [13] $\left(\mathrm{cm}^{-1}\right)$ in emission transition.

$J_{L}: \quad \quad$ Total angular momentum of the lower level.

$g f_{\text {exp }}$ :

$\Delta g f_{\exp }:$

Measured weighted oscillator strength value taken from [13] (dimensionless).

$g f_{\text {calc }}:$

Experimental uncertainty (\%) for measured weighted oscillator strength value from [13].

$\log \left(g f_{\text {calc }}\right): \quad$ Weighted oscillator strength in logarithmic scale from this work (OSP)

Table 3. Comparison between HFR+CPOL radiative lifetimes (in ns) and available experimental data for low-lying $\mathbf{3 d} \mathbf{4}^{\mathbf{4}} \mathbf{5 s}$ energy levels of $\mathbf{C r}$ II.

Level: Designation of the state in LSJ coupling within the configuration and where $J$ represents the total angular momentum.

Energy: $\quad$ Observed (experimental) energy level value in $\mathrm{cm}^{-1}$ [13].

$\tau_{\text {Exp }}: \quad \quad$ Measured lifetimes (ns) obtained by TR-LIF technique [13].

$\tau_{\mathrm{HFR}+\mathrm{CPOL}}$ : $\quad$ Computed lifetime (ns) using Hartree-Fock method with relativistic corrections (HFR) implemented in the code developed by

Table 4. Semi-empirical radiative data in $\mathrm{Cr}$ II

Wavelength:

E(lower):

Wavelength in Å of experimentally observed spectral lines.

Parity (lower):

Energy in $\mathrm{cm}^{-1}$ of lower level of the transition.

$J$ (lower):

Parity of lower level of the transition. 'e' and 'o' stand respectively for even and odd.

E(upper):

Total angular momentum of the lower level of the transition.

Energy in $\mathrm{cm}^{-1}$ of upper level of the transition.

Parity (upper):

$J$ (upper):

Parity of upper level of the transition. 'e' and 'o' stand respectively for even and odd.

$\log (g f)(\mathrm{HFR}+\mathrm{CPOL})$ :

$g A(\mathrm{HFR}+\mathrm{CPOL})$ :

$B F(\mathrm{HFR}+\mathrm{CPOL})$ :

Total angular momentum of the upper level of the transition.

HFR+CPOL weighted oscillator strength in logarithmic scale.

$\quad$ HFR+CPOL branching fraction.

$\log (g f)($ Kurucz):

Weighted oscillator strength in the logarithmic scale from the Kurucz database [14]. 
Table 1

Eigenvalue components of even-parity $\mathrm{Cr}$ II levels of interest in this study, more detailed than in our previous paper [1].

\begin{tabular}{|c|c|c|c|c|c|c|c|c|c|c|}
\hline Level & $J$ & $\begin{array}{l}\text { Energy } \\
\left(\mathrm{cm}^{-1}\right)\end{array}$ & $\begin{array}{l}3 d^{5} \\
(\%)\end{array}$ & $\begin{array}{c}3 d^{4} 4 s \\
(\%)\end{array}$ & $\begin{array}{c}3 \mathrm{~d}^{4} 4 \mathrm{~d} \\
(\%)\end{array}$ & $\begin{array}{c}3 d^{4} 5 s \\
(\%)\end{array}$ & $\begin{array}{c}3 d^{4} 6 s \\
(\%)\end{array}$ & $\begin{array}{c}3 \mathrm{~d}^{4} 5 \mathrm{~d} \\
(\%)\end{array}$ & $\begin{array}{c}3 d^{4} 6 d \\
(\%)\end{array}$ & $\begin{array}{c}\text { Others } \\
(\%)\end{array}$ \\
\hline$e^{6} D$ & $1 / 2$ & 82692.011 & & 0.03 & 0.09 & 99.76 & 0.08 & & & 0.02 \\
\hline$e^{6} D$ & $3 / 2$ & 82763.665 & & 0.03 & 0.09 & 99.76 & 0.08 & & & 0.02 \\
\hline$e^{6} D$ & $5 / 2$ & 82881.097 & & 0.03 & 0.09 & 99.76 & 0.08 & & & 0.02 \\
\hline$e^{6} D$ & $7 / 2$ & 83041.319 & & 0.03 & 0.10 & 99.75 & 0.08 & & & 0.02 \\
\hline$e^{6} D$ & $9 / 2$ & 83240.419 & & 0.03 & 0.10 & 99.75 & 0.08 & & & 0.02 \\
\hline$e^{4} D$ & $1 / 2$ & 84209.784 & 0.03 & 0.09 & 0.02 & 99.85 & & & & \\
\hline$e^{4} D$ & $3 / 2$ & 84320.138 & 0.03 & 0.09 & 0.02 & 99.85 & & & & \\
\hline$e^{4} D$ & $5 / 2$ & 84495.613 & 0.03 & 0.09 & 0.02 & 99.85 & & & & \\
\hline$e^{4} D$ & $7 / 2$ & 84726.677 & 0.03 & 0.09 & 0.02 & 99.85 & & & & \\
\hline$f^{4} P$ & $1 / 2$ & 99677.828 & 0.01 & & & 99.90 & 0.05 & & & 0.01 \\
\hline$f^{4} P$ & $3 / 2$ & 100040.168 & 0.01 & & & 99.89 & 0.05 & & & 0.01 \\
\hline$e^{4} \mathrm{H}$ & $7 / 2$ & 100068.856 & & & 0.02 & 99.89 & 0.06 & & & 0.01 \\
\hline$e^{4} \mathrm{H}$ & $9 / 2$ & 100135.778 & & & 0.02 & 99.89 & 0.06 & & & 0.01 \\
\hline$e^{4} H$ & $11 / 2$ & 100221.595 & & & 0.02 & 99.89 & 0.06 & & & 0.01 \\
\hline$e^{4} \mathrm{H}$ & $13 / 2$ & 100322.077 & & & 0.02 & 99.89 & 0.06 & & & 0.01 \\
\hline$f^{4} P$ & $5 / 2$ & 100650.462 & 0.01 & & & 99.90 & 0.05 & & & 0.01 \\
\hline$e^{2} P$ & $1 / 2$ & 100782.820 & 0.02 & 0.04 & 0.09 & 99.85 & & & & \\
\hline $\mathrm{f}^{4} \mathrm{~F}$ & $3 / 2$ & 101245.003 & & & 0.05 & 99.86 & 0.05 & & & 0.01 \\
\hline $\mathrm{f}^{4} \mathrm{~F}$ & $5 / 2$ & 101276.5471 & & & 0.05 & 99.86 & 0.05 & & & 0.01 \\
\hline$f^{4} F$ & $7 / 2$ & 101321.812 & & & 0.05 & 99.86 & 0.05 & & & 0.01 \\
\hline$e^{2} P$ & $3 / 2$ & 101492.822 & 0.02 & 0.04 & 0.10 & 99.83 & & & & \\
\hline$g^{2} G$ & $7 / 2$ & 104543.110 & 0.03 & 0.03 & 3.04 & 96.83 & & 0.07 & 0.01 & \\
\hline $\mathrm{g}^{2} \mathrm{G}$ & $9 / 2$ & 104666.357 & 0.01 & 0.03 & 1.34 & 98.57 & & 0.03 & & \\
\hline
\end{tabular}

Table 2

Comparison between experimental and calculated oscillator strength values.

\begin{tabular}{|c|c|c|c|c|c|c|c|c|c|}
\hline $\begin{array}{l}\lambda_{\text {air }} \\
(\AA)\end{array}$ & $\begin{array}{l}\text { Upper level } \\
\left(\mathrm{cm}^{-1}\right)\end{array}$ & $J_{U}$ & $\begin{array}{l}\text { Lower level } \\
\quad\left(\mathrm{cm}^{-1}\right)\end{array}$ & $J_{L}$ & $g f_{\text {exp }}$ & $\log \left(g f_{\exp }\right)$ & $\begin{array}{c}\Delta g f_{\exp } \\
(\%)\end{array}$ & $g f_{\text {calc }}$ & $\log \left(g f_{\text {calc }}\right)$ \\
\hline 2039.073 & 82763.664 & $3 / 2$ & 48749.277 & $1 / 2$ & 0.0590 & -1.230 & 19 & 0.0454 & -1.343 \\
\hline 2787.123 & 82692.008 & $1 / 2$ & 46823.305 & $1 / 2$ & 0.2360 & -0.627 & 15 & 0.2377 & -0.624 \\
\hline 2787.914 & 82763.664 & $3 / 2$ & 46905.137 & $3 / 2$ & 0.5020 & -0.299 & 11 & 0.3496 & -0.456 \\
\hline 2789.292 & 82881.094 & $5 / 2$ & 47040.273 & $5 / 2$ & 0.3620 & -0.441 & 12 & 0.4311 & -0.365 \\
\hline 2791.373 & 83041.320 & $7 / 2$ & 47227.219 & $7 / 2$ & 0.5260 & -0.279 & 12 & 0.4278 & -0.369 \\
\hline 2793.497 & 82692.008 & $1 / 2$ & 46905.137 & $3 / 2$ & 0.2070 & -0.684 & 17 & 0.1867 & -0.729 \\
\hline 2794.357 & 83240.422 & $9 / 2$ & 47464.559 & $9 / 2$ & 0.4500 & -0.347 & 13 & 0.3001 & -0.523 \\
\hline 2798.461 & 82763.664 & $3 / 2$ & 47040.273 & $5 / 2$ & 0.2790 & -0.554 & 11 & 0.4288 & -0.368 \\
\hline 2803.918 & 82881.094 & $5 / 2$ & 47227.219 & $7 / 2$ & 0.6930 & -0.159 & 10 & 0.7640 & -0.117 \\
\hline 2809.996 & 83041.320 & $7 / 2$ & 47464.559 & $9 / 2$ & 0.9720 & -0.012 & 9 & 1.2173 & 0.085 \\
\hline 2816.959 & 83240.422 & $9 / 2$ & 47751.602 & $11 / 2$ & 1.3210 & 0.121 & 9 & 1.8215 & 0.260 \\
\hline 2888.628 & 83240.422 & $9 / 2$ & 48632.059 & $7 / 2$ & 1.1590 & 0.064 & 9 & 1.0098 & 0.004 \\
\hline 2893.486 & 83041.320 & $7 / 2$ & 48491.059 & $5 / 2$ & 0.6190 & -0.208 & 9 & 0.5436 & -0.265 \\
\hline 2899.191 & 82881.094 & $5 / 2$ & 48398.871 & $3 / 2$ & 0.5180 & -0.286 & 10 & 0.2138 & -0.670 \\
\hline 2905.344 & 83041.320 & $7 / 2$ & 48632.059 & $7 / 2$ & 0.3210 & -0.493 & 11 & 0.2510 & -0.600 \\
\hline 2906.967 & 82881.094 & $5 / 2$ & 48491.059 & $5 / 2$ & 0.3720 & -0.429 & 10 & 0.3217 & -0.492 \\
\hline 2909.105 & 82763.664 & $3 / 2$ & 48398.871 & $3 / 2$ & 0.3420 & -0.466 & 11 & 0.2574 & -0.589 \\
\hline 2915.182 & 82692.008 & $1 / 2$ & 48398.871 & $3 / 2$ & 0.2110 & -0.676 & 12 & 0.1599 & -0.796 \\
\hline 2916.928 & 82763.664 & $3 / 2$ & 48491.059 & $5 / 2$ & 0.1100 & -0.959 & 16 & 0.0926 & -1.034 \\
\hline 2939.073 & 82763.664 & $3 / 2$ & 48749.277 & $1 / 2$ & 0.0590 & -1.230 & 19 & 0.0454 & -1.343 \\
\hline 2945.275 & 82692.008 & $1 / 2$ & 48749.277 & $1 / 2$ & 0.0510 & -1.290 & 21 & 0.0131 & -1.883 \\
\hline 2951.144 & 82881.094 & $5 / 2$ & 49005.848 & $3 / 2$ & 0.0790 & -1.102 & 17 & 0.0443 & -1.353 \\
\hline 2967.409 & 83041.320 & $7 / 2$ & 49351.734 & $5 / 2$ & 0.2680 & -0.571 & 11 & 0.0775 & -1.110 \\
\hline 2967.728 & 82692.008 & $1 / 2$ & 49005.848 & $3 / 2$ & 0.0930 & -1.032 & 13 & 0.0929 & -1.032 \\
\hline 2975.801 & 83240.422 & $9 / 2$ & 49645.805 & $7 / 2$ & 0.2840 & -0.547 & 10 & 0.1868 & -0.729 \\
\hline 2981.590 & 82881.094 & $5 / 2$ & 49351.734 & $5 / 2$ & 0.1800 & -0.745 & 11 & 0.0944 & -1.025 \\
\hline 2992.070 & 82763.664 & $3 / 2$ & 49351.734 & $5 / 2$ & 0.2930 & -0.533 & 10 & 0.1661 & -0.780 \\
\hline 2992.956 & 83240.422 & $9 / 2$ & 49838.379 & $9 / 2$ & 1.5380 & 0.187 & 9 & 0.9442 & -0.025 \\
\hline 2993.541 & 83041.320 & $7 / 2$ & 49645.805 & $7 / 2$ & 0.5360 & -0.271 & 9 & 0.4334 & -0.363 \\
\hline 2998.970 & 83041.320 & $7 / 2$ & 49706.262 & $5 / 2$ & 0.1850 & -0.733 & 10 & 0.1861 & -0.730 \\
\hline 3000.630 & 82881.094 & $5 / 2$ & 49564.504 & $3 / 2$ & 0.3160 & -0.500 & 10 & 0.2089 & -0.680 \\
\hline 3004.748 & 82763.664 & $3 / 2$ & 49492.711 & $1 / 2$ & 0.1630 & -0.788 & 10 & 0.1394 & -0.856 \\
\hline 3007.973 & 82881.094 & $5 / 2$ & 49645.805 & $7 / 2$ & 0.2810 & -0.551 & 10 & 0.3302 & -0.481 \\
\hline 3010.904 & 83041.320 & $7 / 2$ & 49838.379 & $9 / 2$ & 0.3300 & -0.481 & 10 & 0.2402 & -0.619 \\
\hline 3011.237 & 82692.008 & $1 / 2$ & 49492.711 & $1 / 2$ & 0.0390 & -1.409 & 18 & 0.0407 & -1.391 \\
\hline 3013.471 & 82881.094 & $5 / 2$ & 49706.262 & $5 / 2$ & 0.0316 & -1.500 & 19 & 0.0944 & -1.025 \\
\hline 3017.780 & 82692.008 & $1 / 2$ & 49564.504 & $3 / 2$ & 0.1580 & -0.801 & 12 & 0.1281 & -0.893 \\
\hline 3024.160 & 82763.664 & $3 / 2$ & 49706.262 & $5 / 2$ & 0.0600 & -1.220 & 11 & 0.1505 & -0.823 \\
\hline 3194.194 & 83240.422 & $9 / 2$ & 51942.664 & $9 / 2$ & 0.0520 & -1.284 & 21 & 0.0117 & -1.933 \\
\hline
\end{tabular}


Table 3

Comparison between HFR+CPOL radiative lifetimes (in ns) and available experimental data for low-lying $3 \mathrm{~d}^{4} 5 \mathrm{~s}$ energy levels of $\mathrm{Cr}$ II.

\begin{tabular}{|c|c|c|c|}
\hline Level & $\begin{array}{l}\text { Energy } \\
\left(\mathrm{cm}^{-1}\right)\end{array}$ & $\begin{array}{l}\tau_{\text {Exp }} \\
\text { (ns) }\end{array}$ & $\begin{array}{c}\tau_{H F R+C P O I} \\
\quad(\mathrm{~ns})\end{array}$ \\
\hline$e^{6} D_{1 / 2}$ & 82692.011 & $2.5 \pm 0.2$ & 2.8 \\
\hline$e^{6} D_{3 / 2}$ & 82763.665 & $2.5 \pm 0.2$ & 2.8 \\
\hline$e^{6} D_{5 / 2}$ & 82881.097 & $2.5 \pm 0.2$ & 2.8 \\
\hline$e^{6} D_{7 / 2}$ & 83041.319 & $2.6 \pm 0.2$ & 2.8 \\
\hline$e^{6} D_{9 / 2}$ & 83240.419 & $2.6 \pm 0.2$ & 2.8 \\
\hline$e^{4} D_{1 / 2}$ & 84209.784 & & 2.9 \\
\hline$e^{4} D_{3 / 2}$ & 84320.138 & & 2.9 \\
\hline$e^{4} D_{5 / 2}$ & 84495.613 & & 2.9 \\
\hline$e^{4} D_{7 / 2}$ & 84726.677 & & 2.9 \\
\hline $\mathrm{f}^{4} \mathrm{P}_{1 / 2}$ & 99677.828 & & 2.7 \\
\hline $\mathrm{f}^{4} \mathrm{P}_{3 / 2}$ & 100040.168 & & 2.7 \\
\hline $\mathrm{e}^{4} \mathrm{H}_{7 / 2}$ & 100068.856 & & 2.8 \\
\hline $\mathrm{f}^{4} \mathrm{P}_{5 / 2}$ & 100650.462 & & 2.7 \\
\hline $\mathrm{f}^{2} \mathrm{P}_{1 / 2}$ & 100782.820 & & 2.8 \\
\hline $\mathrm{f}^{4} \mathrm{~F}_{3 / 2}$ & 101245.003 & & 2.7 \\
\hline $\mathrm{f}^{4} \mathrm{~F}_{5 / 2}$ & 101276.547 & & 2.7 \\
\hline $\mathrm{f}^{4} \mathrm{~F}_{7 / 2}$ & 101321.812 & & 2.7 \\
\hline $\mathrm{e}^{2} \mathrm{P}_{3 / 2}$ & 101492.822 & & 2.8 \\
\hline $\mathrm{g}^{2} \mathrm{G}_{7 / 2}$ & 104543.110 & & 2.3 \\
\hline $\mathrm{g}^{2} \mathrm{G}_{9 / 2}$ & 104666.357 & & 2.6 \\
\hline
\end{tabular}


Table 4

Semi-empirical radiative data in $\mathrm{Cr}$ II.

\begin{tabular}{|c|c|c|c|c|c|c|c|c|c|c|}
\hline $\begin{array}{c}\text { Wavelength } \\
\text { (Å) }\end{array}$ & $\begin{array}{c}E \text { (lower) } \\
\left(\mathrm{cm}^{-1}\right)\end{array}$ & Parity (lower) & $J$ (lower) & $\begin{array}{c}E \text { (upper) } \\
\left(\mathrm{cm}^{-1}\right)\end{array}$ & Parity (upper) & $J$ (upper) & $\log (g f)(\mathrm{HFR}+\mathrm{CPOL})$ & $\begin{array}{c}g A(\mathrm{HFR}+\mathrm{CPOL}) \\
\left(\mathrm{s}^{-1}\right)\end{array}$ & $B F(\mathrm{HFR}+\mathrm{CPOL})$ & $\begin{array}{c}\log (g f) \\
\text { (Kurucz) }\end{array}$ \\
\hline 1788.363 & 48749 & (o) & 0.5 & 104666 & (e) & 0.5 & -3.12 & $1.61 \mathrm{E}+06$ & $2.09 \mathrm{E}-03$ & \\
\hline 1796.607 & 49006 & (o) & 1.5 & 104666 & (e) & 0.5 & -3.67 & $4.48 \mathrm{E}+05$ & $5.82 \mathrm{E}-04$ & \\
\hline 1812.460 & 49493 & (o) & 0.5 & 104666 & (e) & 0.5 & -3.67 & $4.34 \mathrm{E}+05$ & $5.64 \mathrm{E}-04$ & \\
\hline 1936.311 & 49006 & (o) & 1.5 & 100650 & (e) & 2.5 & -2.70 & $3.52 \mathrm{E}+06$ & $1.58 \mathrm{E}-03$ & -2.595 \\
\hline 1949.366 & 49352 & (o) & 2.5 & 100650 & (e) & 2.5 & -2.48 & $5.80 \mathrm{E}+06$ & $2.61 \mathrm{E}-03$ & -2.252 \\
\hline 1949.664 & 48749 & (o) & 0.5 & 100040 & (e) & 1.5 & -2.68 & $3.65 E+06$ & $2.46 \mathrm{E}-03$ & -2.597 \\
\hline 1957.485 & 49565 & (o) & 1.5 & 100650 & (e) & 2.5 & -3.12 & $1.34 \mathrm{E}+06$ & $6.03 \mathrm{E}-04$ & -2.964 \\
\hline 1959.466 & 49006 & (o) & 1.5 & 100040 & (e) & 1.5 & -3.24 & $9.98 \mathrm{E}+05$ & $6.74 \mathrm{E}-04$ & -3.107 \\
\hline 1962.932 & 49706 & (o) & 2.5 & 100650 & (e) & 2.5 & -2.51 & $5.39 \mathrm{E}+06$ & $2.43 \mathrm{E}-03$ & -2.492 \\
\hline 1963.534 & 48749 & (o) & 0.5 & 99678 & (e) & 0.5 & -3.34 & $7.96 \mathrm{E}+05$ & $1.07 \mathrm{E}-03$ & -3.296 \\
\hline 1972.837 & 49352 & (o) & 2.5 & 100040 & (e) & 1.5 & -2.79 & $2.79 \mathrm{E}+06$ & $1.88 \mathrm{E}-03$ & -2.619 \\
\hline 1973.476 & 49006 & (o) & 1.5 & 99678 & (e) & 0.5 & -2.68 & $3.58 \mathrm{E}+06$ & $4.83 \mathrm{E}-03$ & -2.611 \\
\hline 1978.339 & 49493 & (o) & 0.5 & 100040 & (e) & 1.5 & -3.22 & $1.03 \mathrm{E}+06$ & $6.95 \mathrm{E}-04$ & -3.003 \\
\hline 1981.153 & 49565 & (o) & 1.5 & 100040 & (e) & 1.5 & -3.64 & $3.86 \mathrm{E}+05$ & $2.61 \mathrm{E}-04$ & -3.489 \\
\hline 1986.732 & 49706 & (o) & 2.5 & 100040 & (e) & 1.5 & -2.83 & $2.52 \mathrm{E}+06$ & $1.70 \mathrm{E}-03$ & -2.778 \\
\hline 1992.622 & 49493 & (o) & 0.5 & 99678 & (e) & 0.5 & -3.88 & $2.23 \mathrm{E}+05$ & $3.01 \mathrm{E}-04$ & -3.654 \\
\hline 1995.476 & 49565 & (o) & 1.5 & 99678 & (e) & 0.5 & -3.10 & $1.35 \mathrm{E}+06$ & $1.82 \mathrm{E}-03$ & -2.912 \\
\hline 2011.728 & 51584 & (o) & 1.5 & 101277 & (e) & 2.5 & -3.21 & $1.03 \mathrm{E}+06$ & $4.64 \mathrm{E}-04$ & -3.003 \\
\hline 2013.006 & 51584 & (o) & 1.5 & 101245 & (e) & 1.5 & -2.44 & $5.99 \mathrm{E}+06$ & $4.04 \mathrm{E}-03$ & -2.262 \\
\hline 2013.351 & 51669 & (o) & 2.5 & 101322 & (e) & 3.5 & -3.13 & $1.22 \mathrm{E}+06$ & $4.12 \mathrm{E}-04$ & -2.926 \\
\hline 2015.188 & 51669 & (o) & 2.5 & 101277 & (e) & 2.5 & -2.30 & $8.36 \mathrm{E}+06$ & $3.76 \mathrm{E}-03$ & -2.129 \\
\hline 2016.470 & 51669 & (o) & 2.5 & 101245 & (e) & 1.5 & -3.11 & $1.28 \mathrm{E}+06$ & $8.64 \mathrm{E}-04$ & -2.878 \\
\hline 2020.051 & 51789 & (o) & 3.5 & 101277 & (e) & 2.5 & -2.99 & $1.67 \mathrm{E}+06$ & $7.52 \mathrm{E}-04$ & -2.751 \\
\hline 2172.058 & 54626 & (o) & 2.5 & 100650 & (e) & 2.5 & -3.24 & $8.18 \mathrm{E}+05$ & $3.68 \mathrm{E}-04$ & -2.977 \\
\hline 2179.581 & 54784 & (o) & 3.5 & 100650 & (e) & 2.5 & -2.76 & $2.43 \mathrm{E}+06$ & $1.09 \mathrm{E}-03$ & -2.422 \\
\hline 2191.230 & 54418 & (o) & 0.5 & 100040 & (e) & 1.5 & -3.89 & $1.78 \mathrm{E}+05$ & $1.20 \mathrm{E}-04$ & -3.702 \\
\hline 2195.153 & 54499 & (o) & 1.5 & 100040 & (e) & 1.5 & -3.18 & $9.17 \mathrm{E}+05$ & $6.19 \mathrm{E}-04$ & -2.935 \\
\hline 2201.249 & 54626 & (o) & 2.5 & 100040 & (e) & 1.5 & -3.07 & $1.17 \mathrm{E}+06$ & $7.90 \mathrm{E}-04$ & -2.72 \\
\hline 2208.773 & 54418 & (o) & 0.5 & 99678 & (e) & 0.5 & -3.30 & $6.88 \mathrm{E}+05$ & $9.29 \mathrm{E}-04$ & -3.053 \\
\hline 2212.760 & 54499 & (o) & 1.5 & 99678 & (e) & 0.5 & -3.46 & $4.77 \mathrm{E}+05$ & $6.44 \mathrm{E}-04$ & -3.106 \\
\hline 2446.365 & 63802 & (o) & 0.5 & 104666 & (e) & 0.5 & -1.93 & $1.33 \mathrm{E}+07$ & $1.73 \mathrm{E}-02$ & \\
\hline 2462.027 & 64062 & (o) & 1.5 & 104666 & (e) & 0.5 & -2.00 & $1.12 \mathrm{E}+07$ & $1.46 \mathrm{E}-02$ & \\
\hline 2493.364 & 64449 & (o) & 2.5 & 104543 & (e) & 3.5 & -2.03 & $1.01 \mathrm{E}+07$ & $2.90 \mathrm{E}-03$ & -2.181 \\
\hline 2522.136 & 65029 & (o) & 0.5 & 104666 & (e) & 0.5 & -0.85 & $1.48 \mathrm{E}+08$ & $1.92 \mathrm{E}-01$ & \\
\hline 2602.718 & 66256 & (o) & 0.5 & 104666 & (e) & 0.5 & -1.20 & $6.30 \mathrm{E}+07$ & $8.19 \mathrm{E}-02$ & \\
\hline 2609.398 & 66355 & (o) & 1.5 & 104666 & (e) & 0.5 & -2.60 & $2.51 \mathrm{E}+06$ & $3.26 \mathrm{E}-03$ & \\
\hline 2629.622 & 66649 & (o) & 1.5 & 104666 & (e) & 0.5 & -0.68 & $2.03 \mathrm{E}+08$ & $2.64 \mathrm{E}-01$ & \\
\hline 2643.573 & 66727 & (o) & 2.5 & 104543 & (e) & 3.5 & -2.30 & $4.83 \mathrm{E}+06$ & $1.39 \mathrm{E}-03$ & -2.596 \\
\hline 2645.099 & 66872 & (o) & 0.5 & 104666 & (e) & 0.5 & -0.41 & $3.77 \mathrm{E}+08$ & $4.90 \mathrm{E}-01$ & \\
\hline 2652.359 & 63802 & (o) & 0.5 & 101493 & (e) & 1.5 & -3.60 & $2.37 \mathrm{E}+05$ & $1.66 \mathrm{E}-04$ & -3.786 \\
\hline 2653.442 & 63601 & (o) & 3.5 & 101277 & (e) & 2.5 & -2.01 & $9.28 \mathrm{E}+06$ & $4.18 \mathrm{E}-03$ & -2.214 \\
\hline 2659.073 & 67070 & (o) & 1.5 & 104666 & (e) & 0.5 & -3.61 & $2.35 \mathrm{E}+05$ & $3.06 \mathrm{E}-04$ & \\
\hline 2663.669 & 67012 & (o) & 2.5 & 104543 & (e) & 3.5 & -2.74 & $1.72 \mathrm{E}+06$ & $4.95 \mathrm{E}-04$ & -2.408 \\
\hline 2669.053 & 47040 & (o) & 2.5 & 84496 & (e) & 2.5 & -3.35 & $4.21 \mathrm{E}+05$ & $2.03 \mathrm{E}-04$ & -3.308 \\
\hline 2669.914 & 63802 & (o) & 0.5 & 101245 & (e) & 1.5 & -1.43 & $3.47 \mathrm{E}+07$ & $2.34 \mathrm{E}-02$ & -1.6 \\
\hline 2670.779 & 64062 & (o) & 1.5 & 101493 & (e) & 1.5 & -2.83 & $1.40 \mathrm{E}+06$ & $9.80 \mathrm{E}-04$ & -2.76 \\
\hline 2671.930 & 46905 & (o) & 1.5 & 84320 & (e) & 1.5 & -3.85 & $1.32 \mathrm{E}+05$ & $9.57 \mathrm{E}-05$ & -3.882 \\
\hline 2679.835 & 46905 & (o) & 1.5 & 84210 & (e) & 0.5 & -3.08 & $7.81 \mathrm{E}+05$ & $1.13 \mathrm{E}-03$ & -2.896 \\
\hline 2681.100 & 67379 & (o) & 1.5 & 104666 & (e) & 0.5 & -1.45 & $3.32 \mathrm{E}+07$ & $4.32 \mathrm{E}-02$ & \\
\hline 2681.617 & 47040 & (o) & 2.5 & 84320 & (e) & 1.5 & -2.58 & $2.45 \mathrm{E}+06$ & $1.78 \mathrm{E}-03$ & -2.417 \\
\hline 2682.442 & 47227 & (o) & 3.5 & 84496 & (e) & 2.5 & -2.31 & $4.53 \mathrm{E}+06$ & $2.19 \mathrm{E}-03$ & -2.152 \\
\hline 2686.301 & 64062 & (o) & 1.5 & 101277 & (e) & 2.5 & -1.18 & $6.15 \mathrm{E}+07$ & $2.77 \mathrm{E}-02$ & -1.317 \\
\hline 2687.439 & 67344 & (o) & 2.5 & 104543 & (e) & 3.5 & -1.95 & $1.04 \mathrm{E}+07$ & $2.99 \mathrm{E}-03$ & -2.09 \\
\hline 2688.580 & 64062 & (o) & 1.5 & 101245 & (e) & 1.5 & -1.84 & $1.34 \mathrm{E}+07$ & $9.05 \mathrm{E}-03$ & -2.02 \\
\hline 2690.555 & 67387 & (o) & 2.5 & 104543 & (e) & 3.5 & -3.87 & $1.24 \mathrm{E}+05$ & $3.57 \mathrm{E}-05$ & -3.369 \\
\hline 2698.687 & 64449 & (o) & 2.5 & 101493 & (e) & 1.5 & -1.83 & $1.37 \mathrm{E}+07$ & $9.59 \mathrm{E}-03$ & -1.71 \\
\hline 2711.203 & 64449 & (o) & 2.5 & 101322 & (e) & 3.5 & -0.93 & $1.09 \mathrm{E}+08$ & $3.68 \mathrm{E}-02$ & -1.027 \\
\hline 2714.536 & 64449 & (o) & 2.5 & 101277 & (e) & 2.5 & -1.68 & $1.91 \mathrm{E}+07$ & $8.60 \mathrm{E}-03$ & -1.841 \\
\hline 2716.086 & 67860 & (o) & 0.5 & 104666 & (e) & 0.5 & -2.79 & $1.47 \mathrm{E}+06$ & $1.91 \mathrm{E}-03$ & \\
\hline 2716.863 & 64449 & (o) & 2.5 & 101245 & (e) & 1.5 & -2.93 & $1.06 \mathrm{E}+06$ & $7.16 \mathrm{E}-04$ & -3.118 \\
\hline 2716.872 & 67870 & (o) & 1.5 & 104666 & (e) & 0.5 & -3.81 & $1.41 \mathrm{E}+05$ & $1.83 \mathrm{E}-04$ & \\
\hline 2722.421 & 64062 & (o) & 1.5 & 100783 & (e) & 0.5 & -2.00 & $9.04 \mathrm{E}+06$ & $1.27 \mathrm{E}-02$ & -1.923 \\
\hline 2725.822 & 67868 & (o) & 2.5 & 104543 & (e) & 3.5 & -1.47 & $3.02 \mathrm{E}+07$ & $8.68 \mathrm{E}-03$ & -3.563 \\
\hline 2732.270 & 64062 & (o) & 1.5 & 100650 & (e) & 2.5 & -1.76 & $1.57 \mathrm{E}+07$ & $7.07 \mathrm{E}-03$ & -1.659 \\
\hline 2741.659 & 65029 & (o) & 0.5 & 101493 & (e) & 1.5 & -1.07 & $7.62 \mathrm{E}+07$ & $5.33 \mathrm{E}-02$ & -1.043 \\
\hline
\end{tabular}


Table 4 (continued)

\begin{tabular}{|c|c|c|c|c|c|c|c|c|c|c|}
\hline $\begin{array}{c}\text { Wavelength } \\
(\AA)\end{array}$ & $\begin{array}{c}E \text { (lower) } \\
\left(\mathrm{cm}^{-1}\right)\end{array}$ & Parity (lower) & $J($ lower) & $\begin{array}{c}E \text { (upper) } \\
\left(\mathrm{cm}^{-1}\right)\end{array}$ & Parity (upper) & $J$ (upper) & $\log (g f)(\mathrm{HFR}+\mathrm{CPOL})$ & $\begin{array}{c}g A(\mathrm{HFR}+\mathrm{CPOL}) \\
\left(\mathrm{s}^{-1}\right)\end{array}$ & $B F(\mathrm{HFR}+\mathrm{CPOL})$ & $\begin{array}{c}\log (g f) \\
\text { (Kurucz) }\end{array}$ \\
\hline 2749.403 & 68306 & (o) & 1.5 & 104666 & (e) & 0.5 & -3.19 & $5.76 \mathrm{E}+05$ & $7.49 \mathrm{E}-04$ & \\
\hline 2750.061 & 64924 & (o) & 3.5 & 101277 & (e) & 2.5 & -3.11 & $6.85 \mathrm{E}+05$ & $3.08 \mathrm{E}-04$ & -3.287 \\
\hline 2758.687 & 63802 & (o) & 0.5 & 100040 & (e) & 1.5 & -1.45 & $3.12 \mathrm{E}+07$ & $2.11 \mathrm{E}-02$ & -1.411 \\
\hline 2760.420 & 65029 & (o) & 0.5 & 101245 & (e) & 1.5 & -3.42 & $3.33 \mathrm{E}+05$ & $2.25 \mathrm{E}-04$ & -3.849 \\
\hline 2761.485 & 64449 & (o) & 2.5 & 100650 & (e) & 2.5 & -0.74 & $1.61 \mathrm{E}+08$ & $7.25 \mathrm{E}-02$ & -0.681 \\
\hline 2764.263 & 65156 & (o) & 2.5 & 101322 & (e) & 3.5 & -1.99 & $8.87 \mathrm{E}+06$ & $2.99 \mathrm{E}-03$ & -1.879 \\
\hline 2767.728 & 65156 & (o) & 2.5 & 101277 & (e) & 2.5 & -0.84 & $1.26 \mathrm{E}+08$ & $5.67 \mathrm{E}-02$ & -0.856 \\
\hline 2769.515 & 48399 & (o) & 1.5 & 84496 & (e) & 2.5 & -2.79 & $1.42 \mathrm{E}+06$ & $6.86 \mathrm{E}-04$ & -2.044 \\
\hline 2770.147 & 65156 & (o) & 2.5 & 101245 & (e) & 1.5 & -0.27 & $4.66 \mathrm{E}+08$ & $3.15 \mathrm{E}-01$ & -0.288 \\
\hline 2775.440 & 65257 & (o) & 3.5 & 101277 & (e) & 2.5 & -0.44 & $3.10 \mathrm{E}+08$ & $1.40 \mathrm{E}-01$ & -0.389 \\
\hline 2776.607 & 48491 & (o) & 2.5 & 84496 & (e) & 2.5 & -3.05 & $7.86 \mathrm{E}+05$ & $3.80 \mathrm{E}-04$ & -2.361 \\
\hline 2776.878 & 47040 & (o) & 2.5 & 83041 & (e) & 3.5 & -1.29 & $4.47 \mathrm{E}+07$ & $1.12 \mathrm{E}-02$ & -1.257 \\
\hline 2778.619 & 64062 & (o) & 1.5 & 100040 & (e) & 1.5 & -0.59 & $2.26 \mathrm{E}+08$ & $1.53 \mathrm{E}-01$ & -0.582 \\
\hline 2778.814 & 46905 & (o) & 1.5 & 82881 & (e) & 2.5 & -1.14 & $6.22 \mathrm{E}+07$ & $2.07 \mathrm{E}-02$ & -1.132 \\
\hline 2780.063 & 68583 & (o) & 2.5 & 104543 & (e) & 3.5 & -0.21 & $5.28 \mathrm{E}+08$ & $1.52 \mathrm{E}-01$ & -1.414 \\
\hline 2781.566 & 46823 & (o) & 0.5 & 82764 & (e) & 1.5 & -1.18 & $5.74 \mathrm{E}+07$ & $2.87 \mathrm{E}-02$ & -1.182 \\
\hline 2783.045 & 48399 & (o) & 1.5 & 84320 & (e) & 1.5 & -3.00 & $8.75 \mathrm{E}+05$ & $6.34 \mathrm{E}-04$ & -2.215 \\
\hline 2786.549 & 63802 & (o) & 0.5 & 99678 & (e) & 0.5 & -0.64 & $1.98 \mathrm{E}+08$ & $2.67 \mathrm{E}-01$ & -0.669 \\
\hline 2787.123 & 46823 & (o) & 0.5 & 82692 & (e) & 0.5 & -0.63 & $2.00 \mathrm{E}+08$ & $2.00 \mathrm{E}-01$ & -0.657 \\
\hline 2787.914 & 46905 & (o) & 1.5 & 82764 & (e) & 1.5 & -0.47 & $2.92 \mathrm{E}+08$ & $1.46 \mathrm{E}-01$ & -0.486 \\
\hline 2789.292 & 47040 & (o) & 2.5 & 82881 & (e) & 2.5 & -0.38 & $3.57 \mathrm{E}+08$ & $1.19 \mathrm{E}-01$ & -0.39 \\
\hline 2790.206 & 48491 & (o) & 2.5 & 84320 & (e) & 1.5 & -3.86 & $1.21 \mathrm{E}+05$ & $8.77 \mathrm{E}-05$ & -3.194 \\
\hline 2791.622 & 48399 & (o) & 1.5 & 84210 & (e) & 0.5 & -3.76 & $1.52 \mathrm{E}+05$ & $2.20 \mathrm{E}-04$ & -2.967 \\
\hline 2793.497 & 46905 & (o) & 1.5 & 82692 & (e) & 0.5 & -0.73 & $1.58 \mathrm{E}+08$ & $1.58 \mathrm{E}-01$ & -0.769 \\
\hline 2796.106 & 65029 & (o) & 0.5 & 100783 & (e) & 0.5 & -0.84 & $1.25 \mathrm{E}+08$ & $1.75 \mathrm{E}-01$ & -0.903 \\
\hline 2797.658 & 65543 & (o) & 3.5 & 101277 & (e) & 2.5 & -0.51 & $2.61 \mathrm{E}+08$ & $1.17 \mathrm{E}-01$ & -0.649 \\
\hline 2798.257 & 64924 & (o) & 3.5 & 100650 & (e) & 2.5 & -0.02 & $8.15 \mathrm{E}+08$ & $3.67 \mathrm{E}-01$ & -0.043 \\
\hline 2798.461 & 47040 & (o) & 2.5 & 82764 & (e) & 1.5 & -0.37 & $3.63 \mathrm{E}+08$ & $1.82 \mathrm{E}-01$ & -0.409 \\
\hline 2803.918 & 47227 & (o) & 3.5 & 82881 & (e) & 2.5 & -0.12 & $6.43 \mathrm{E}+08$ & $2.14 \mathrm{E}-01$ & -0.158 \\
\hline 2806.887 & 64062 & (o) & 1.5 & 99678 & (e) & 0.5 & -0.63 & $1.98 \mathrm{E}+08$ & $2.67 \mathrm{E}-01$ & -0.698 \\
\hline 2808.838 & 64449 & (o) & 2.5 & 100040 & (e) & 1.5 & -0.25 & $4.76 \mathrm{E}+08$ & $3.21 \mathrm{E}-01$ & -0.307 \\
\hline 2810.462 & 48749 & (o) & 0.5 & 84320 & (e) & 1.5 & -0.97 & $9.04 \mathrm{E}+07$ & $6.55 \mathrm{E}-02$ & -1.006 \\
\hline 2816.884 & 49006 & (o) & 1.5 & 84496 & (e) & 2.5 & -0.61 & $2.06 \mathrm{E}+08$ & $9.96 \mathrm{E}-02$ & -0.643 \\
\hline 2819.209 & 48749 & (o) & 0.5 & 84210 & (e) & 0.5 & -0.93 & $9.88 \mathrm{E}+07$ & $1.43 \mathrm{E}-01$ & -0.96 \\
\hline 2824.538 & 65257 & (o) & 3.5 & 100650 & (e) & 2.5 & -3.53 & $2.44 \mathrm{E}+05$ & $1.10 \mathrm{E}-04$ & \\
\hline 2830.569 & 69348 & (o) & 1.5 & 104666 & (e) & 0.5 & -0.73 & $1.60 \mathrm{E}+08$ & $2.08 \mathrm{E}-01$ & \\
\hline 2830.882 & 49006 & (o) & 1.5 & 84320 & (e) & 1.5 & -0.83 & $1.24 \mathrm{E}+08$ & $8.99 \mathrm{E}-02$ & -0.856 \\
\hline 2837.142 & 66256 & (o) & 0.5 & 101493 & (e) & 1.5 & -2.04 & $7.56 \mathrm{E}+06$ & $5.29 \mathrm{E}-03$ & -1.814 \\
\hline 2839.756 & 49006 & (o) & 1.5 & 84210 & (e) & 0.5 & -1.61 & $2.02 \mathrm{E}+07$ & $2.93 \mathrm{E}-02$ & -1.635 \\
\hline 2844.609 & 49352 & (o) & 2.5 & 84496 & (e) & 2.5 & -0.97 & $8.80 \mathrm{E}+07$ & $4.25 \mathrm{E}-02$ & -0.917 \\
\hline 2845.080 & 66355 & (o) & 1.5 & 101493 & (e) & 1.5 & -3.59 & $2.15 \mathrm{E}+05$ & $1.51 \mathrm{E}-04$ & -2.06 \\
\hline 2847.553 & 65543 & (o) & 3.5 & 100650 & (e) & 2.5 & -3.27 & $4.37 \mathrm{E}+05$ & $1.97 \mathrm{E}-04$ & -3.612 \\
\hline 2850.992 & 69478 & (o) & 2.5 & 104543 & (e) & 3.5 & -1.74 & $1.49 \mathrm{E}+07$ & $4.28 \mathrm{E}-03$ & -2.579 \\
\hline 2854.041 & 69639 & (o) & 1.5 & 104666 & (e) & 0.5 & -2.29 & $4.19 \mathrm{E}+06$ & $5.45 \mathrm{E}-03$ & \\
\hline 2855.420 & 65029 & (o) & 0.5 & 100040 & (e) & 1.5 & -1.37 & $3.47 \mathrm{E}+07$ & $2.34 \mathrm{E}-02$ & -1.428 \\
\hline 2858.885 & 49352 & (o) & 2.5 & 84320 & (e) & 1.5 & -1.99 & $8.39 \mathrm{E}+06$ & $6.08 \mathrm{E}-03$ & -1.889 \\
\hline 2861.937 & 49565 & (o) & 1.5 & 84496 & (e) & 2.5 & -0.98 & $8.45 \mathrm{E}+07$ & $4.08 \mathrm{E}-02$ & -1.041 \\
\hline 2862.701 & 66355 & (o) & 1.5 & 101277 & (e) & 2.5 & -2.72 & $1.57 \mathrm{E}+06$ & $7.07 \mathrm{E}-04$ & -3.097 \\
\hline 2863.474 & 65156 & (o) & 2.5 & 100069 & (e) & 3.5 & -1.24 & $4.56 \mathrm{E}+07$ & $1.60 \mathrm{E}-02$ & -1.373 \\
\hline 2868.614 & 49646 & (o) & 3.5 & 84496 & (e) & 2.5 & -2.68 & $1.70 \mathrm{E}+06$ & $8.22 \mathrm{E}-04$ & -2.738 \\
\hline 2869.139 & 66649 & (o) & 1.5 & 101493 & (e) & 1.5 & -0.84 & $1.16 \mathrm{E}+08$ & $8.12 \mathrm{E}-02$ & -0.591 \\
\hline 2870.458 & 49493 & (o) & 0.5 & 84320 & (e) & 1.5 & -1.48 & $2.70 \mathrm{E}+07$ & $1.96 \mathrm{E}-02$ & -1.486 \\
\hline 2873.599 & 49706 & (o) & 2.5 & 84496 & (e) & 2.5 & -1.17 & $5.52 \mathrm{E}+07$ & $2.67 \mathrm{E}-02$ & -1.395 \\
\hline 2875.527 & 66727 & (o) & 2.5 & 101493 & (e) & 1.5 & -2.21 & $4.96 \mathrm{E}+06$ & $3.47 \mathrm{E}-03$ & -2.392 \\
\hline 2876.387 & 49565 & (o) & 1.5 & 84320 & (e) & 1.5 & -1.32 & $3.83 \mathrm{E}+07$ & $2.78 \mathrm{E}-02$ & -1.398 \\
\hline 2879.582 & 49493 & (o) & 0.5 & 84210 & (e) & 0.5 & -1.49 & $2.58 \mathrm{E}+07$ & $3.74 \mathrm{E}-02$ & -1.513 \\
\hline 2885.280 & 65029 & (o) & 0.5 & 99678 & (e) & 0.5 & -2.15 & $5.72 \mathrm{E}+06$ & $7.72 \mathrm{E}-03$ & -2.131 \\
\hline 2885.550 & 49565 & (o) & 1.5 & 84210 & (e) & 0.5 & -2.05 & $7.14 \mathrm{E}+06$ & $1.04 \mathrm{E}-02$ & -2.138 \\
\hline 2887.060 & 66649 & (o) & 1.5 & 101277 & (e) & 2.5 & -2.43 & $2.98 \mathrm{E}+06$ & $1.34 \mathrm{E}-03$ & -2.696 \\
\hline 2887.574 & 66872 & (o) & 0.5 & 101493 & (e) & 1.5 & -0.73 & $1.49 \mathrm{E}+08$ & $1.04 \mathrm{E}-01$ & -0.803 \\
\hline 2888.168 & 49706 & (o) & 2.5 & 84320 & (e) & 1.5 & -1.90 & $9.99 \mathrm{E}+06$ & $7.24 \mathrm{E}-03$ & -2.099 \\
\hline 2889.693 & 66649 & (o) & 1.5 & 101245 & (e) & 1.5 & -1.63 & $1.89 \mathrm{E}+07$ & $1.28 \mathrm{E}-02$ & -1.842 \\
\hline 2889.741 & 66727 & (o) & 2.5 & 101322 & (e) & 3.5 & -2.02 & $7.78 \mathrm{E}+06$ & $2.63 \mathrm{E}-03$ & -2.377 \\
\hline 2890.240 & 69954 & (o) & 2.5 & 104543 & (e) & 3.5 & -2.36 & $3.53 \mathrm{E}+06$ & $1.01 \mathrm{E}-03$ & -2.927 \\
\hline 2893.486 & 48491 & (o) & 2.5 & 83041 & (e) & 3.5 & -0.28 & $4.26 \mathrm{E}+08$ & $1.07 \mathrm{E}-01$ & -0.239 \\
\hline 2895.488 & 66256 & (o) & 0.5 & 100783 & (e) & 0.5 & -1.41 & $3.13 \mathrm{E}+07$ & $4.38 \mathrm{E}-02$ & -1.585 \\
\hline
\end{tabular}


Table 4 (continued)

\begin{tabular}{|c|c|c|c|c|c|c|c|c|c|c|}
\hline $\begin{array}{c}\text { Wavelength } \\
(\AA)\end{array}$ & $\begin{array}{c}E \text { (lower) } \\
\left(\mathrm{cm}^{-1}\right)\end{array}$ & Parity (lower) & $J$ (lower) & $\begin{array}{c}E \text { (upper) } \\
\left(\mathrm{cm}^{-1}\right)\end{array}$ & Parity (upper) & $J$ (upper) & $\log (g f)(\mathrm{HFR}+\mathrm{CPOL})$ & $\begin{array}{c}g A(\mathrm{HFR}+\mathrm{CPOL}) \\
\left(\mathrm{s}^{-1}\right)\end{array}$ & $B F(\mathrm{HFR}+\mathrm{CPOL})$ & $\begin{array}{c}\log (g f) \\
\text { (Kurucz) }\end{array}$ \\
\hline 2896.172 & 66727 & (o) & 2.5 & 101245 & (e) & 1.5 & -3.79 & $1.30 \mathrm{E}+05$ & $8.78 \mathrm{E}-05$ & -3.48 \\
\hline 2899.195 & 48399 & (o) & 1.5 & 82881 & (e) & 2.5 & -0.70 & $1.59 \mathrm{E}+08$ & $5.30 \mathrm{E}-02$ & -0.638 \\
\hline 2899.320 & 67012 & (o) & 2.5 & 101493 & (e) & 1.5 & -1.20 & $5.02 \mathrm{E}+07$ & $3.51 \mathrm{E}-02$ & -1.326 \\
\hline 2903.756 & 66355 & (o) & 1.5 & 100783 & (e) & 0.5 & -2.83 & $1.17 \mathrm{E}+06$ & $1.64 \mathrm{E}-03$ & -2.659 \\
\hline 2904.236 & 67070 & (o) & 1.5 & 101493 & (e) & 1.5 & -0.65 & $1.79 \mathrm{E}+08$ & $1.25 \mathrm{E}-01$ & -0.901 \\
\hline 2906.967 & 48491 & (o) & 2.5 & 82881 & (e) & 2.5 & -0.45 & $2.84 \mathrm{E}+08$ & $9.47 \mathrm{E}-02$ & -0.512 \\
\hline 2908.394 & 66872 & (o) & 0.5 & 101245 & (e) & 1.5 & -2.48 & $2.66 \mathrm{E}+06$ & $1.80 \mathrm{E}-03$ & -2.522 \\
\hline 2909.103 & 48399 & (o) & 1.5 & 82764 & (e) & 1.5 & -0.56 & $2.20 \mathrm{E}+08$ & $1.10 \mathrm{E}-01$ & -0.593 \\
\hline 2913.771 & 67012 & (o) & 2.5 & 101322 & (e) & 3.5 & -1.01 & $7.57 \mathrm{E}+07$ & $2.55 \mathrm{E}-02$ & -0.845 \\
\hline 2914.964 & 66355 & (o) & 1.5 & 100650 & (e) & 2.5 & -0.78 & $1.32 \mathrm{E}+08$ & $5.94 \mathrm{E}-02$ & -0.638 \\
\hline 2915.181 & 48399 & (o) & 1.5 & 82692 & (e) & 0.5 & -0.72 & $1.51 \mathrm{E}+08$ & $1.51 \mathrm{E}-01$ & -0.832 \\
\hline 2916.928 & 48491 & (o) & 2.5 & 82764 & (e) & 1.5 & -0.95 & $8.99 \mathrm{E}+07$ & $4.50 \mathrm{E}-02$ & -1.102 \\
\hline 2917.621 & 67012 & (o) & 2.5 & 101277 & (e) & 2.5 & -0.77 & $1.33 \mathrm{E}+08$ & $5.99 \mathrm{E}-02$ & -0.8 \\
\hline 2918.935 & 48632 & (o) & 3.5 & 82881 & (e) & 2.5 & -1.33 & $3.70 \mathrm{E}+07$ & $1.23 \mathrm{E}-02$ & -1.52 \\
\hline 2920.309 & 67012 & (o) & 2.5 & 101245 & (e) & 1.5 & -0.86 & $1.07 \mathrm{E}+08$ & $7.22 \mathrm{E}-02$ & -1.159 \\
\hline 2920.875 & 70317 & (o) & 2.5 & 104543 & (e) & 3.5 & -1.76 & $1.37 \mathrm{E}+07$ & $3.94 \mathrm{E}-03$ & -2.366 \\
\hline 2922.599 & 67070 & (o) & 1.5 & 101277 & (e) & 2.5 & -1.18 & $5.20 \mathrm{E}+07$ & $2.34 \mathrm{E}-02$ & -1.114 \\
\hline 2925.297 & 67070 & (o) & 1.5 & 101245 & (e) & 1.5 & -0.97 & $8.35 \mathrm{E}+07$ & $5.64 \mathrm{E}-02$ & -0.97 \\
\hline 2927.503 & 67344 & (o) & 2.5 & 101493 & (e) & 1.5 & -1.87 & $1.07 \mathrm{E}+07$ & $7.49 \mathrm{E}-03$ & \\
\hline 2928.822 & 66649 & (o) & 1.5 & 100783 & (e) & 0.5 & -0.67 & $1.64 \mathrm{E}+08$ & $2.30 \mathrm{E}-01$ & -0.801 \\
\hline 2930.531 & 67379 & (o) & 1.5 & 101493 & (e) & 1.5 & -1.25 & $4.36 \mathrm{E}+07$ & $3.05 \mathrm{E}-02$ & -1.513 \\
\hline 2931.201 & 67387 & (o) & 2.5 & 101493 & (e) & 1.5 & -1.08 & $6.55 \mathrm{E}+07$ & $4.59 \mathrm{E}-02$ & -1.163 \\
\hline 2939.073 & 48749 & (o) & 0.5 & 82764 & (e) & 1.5 & -1.31 & $3.77 \mathrm{E}+07$ & $1.89 \mathrm{E}-02$ & -1.334 \\
\hline 2940.224 & 66649 & (o) & 1.5 & 100650 & (e) & 2.5 & -1.44 & $2.77 \mathrm{E}+07$ & $1.25 \mathrm{E}-02$ & -1.839 \\
\hline 2942.237 & 67344 & (o) & 2.5 & 101322 & (e) & 3.5 & -2.04 & $7.15 \mathrm{E}+06$ & $2.41 \mathrm{E}-03$ & -2.358 \\
\hline 2943.891 & 70584 & (o) & 2.5 & 104543 & (e) & 3.5 & -0.87 & $1.05 \mathrm{E}+08$ & $3.02 \mathrm{E}-02$ & -0.49 \\
\hline 2945.274 & 67334 & (o) & 3.5 & 101277 & (e) & 2.5 & -0.36 & $3.39 \mathrm{E}+08$ & $1.53 \mathrm{E}-01$ & -0.88 \\
\hline 2945.277 & 48749 & (o) & 0.5 & 82692 & (e) & 0.5 & -1.86 & $1.06 \mathrm{E}+07$ & $1.06 \mathrm{E}-02$ & -1.861 \\
\hline 2945.973 & 67387 & (o) & 2.5 & 101322 & (e) & 3.5 & -1.12 & $5.91 \mathrm{E}+07$ & $1.99 \mathrm{E}-02$ & -1.024 \\
\hline 2946.162 & 67344 & (o) & 2.5 & 101277 & (e) & 2.5 & -1.36 & $3.39 \mathrm{E}+07$ & $1.53 \mathrm{E}-02$ & -1.341 \\
\hline 2946.932 & 66727 & (o) & 2.5 & 100650 & (e) & 2.5 & -0.21 & $4.77 \mathrm{E}+08$ & $2.15 \mathrm{E}-01$ & -0.261 \\
\hline 2948.035 & 66872 & (o) & 0.5 & 100783 & (e) & 0.5 & -1.00 & $7.65 \mathrm{E}+07$ & $1.07 \mathrm{E}-01$ & -0.918 \\
\hline 2948.904 & 67344 & (o) & 2.5 & 101245 & (e) & 1.5 & -0.98 & $8.10 \mathrm{E}+07$ & $5.47 \mathrm{E}-02$ & -0.963 \\
\hline 2949.230 & 67379 & (o) & 1.5 & 101277 & (e) & 2.5 & -1.19 & $4.98 \mathrm{E}+07$ & $2.24 \mathrm{E}-02$ & -1.108 \\
\hline 2949.908 & 67387 & (o) & 2.5 & 101277 & (e) & 2.5 & -0.47 & $2.59 \mathrm{E}+08$ & $1.17 \mathrm{E}-01$ & -0.58 \\
\hline 2950.462 & 67393 & (o) & 3.5 & 101277 & (e) & 2.5 & -1.87 & $1.04 \mathrm{E}+07$ & $4.68 \mathrm{E}-03$ & -0.568 \\
\hline 2951.145 & 49006 & (o) & 1.5 & 82881 & (e) & 2.5 & -1.13 & $5.68 \mathrm{E}+07$ & $1.89 \mathrm{E}-02$ & -1.473 \\
\hline 2951.977 & 67379 & (o) & 1.5 & 101245 & (e) & 1.5 & -0.49 & $2.48 \mathrm{E}+08$ & $1.67 \mathrm{E}-01$ & -0.553 \\
\hline 2952.656 & 67387 & (o) & 2.5 & 101245 & (e) & 1.5 & -1.18 & $5.13 \mathrm{E}+07$ & $3.46 \mathrm{E}-02$ & -1.002 \\
\hline 2959.140 & 66256 & (o) & 0.5 & 100040 & (e) & 1.5 & -0.66 & $1.69 \mathrm{E}+08$ & $1.14 \mathrm{E}-01$ & -0.691 \\
\hline 2961.412 & 49006 & (o) & 1.5 & 82764 & (e) & 1.5 & -2.21 & $4.71 \mathrm{E}+06$ & $2.36 \mathrm{E}-03$ & -1.713 \\
\hline 2965.403 & 67070 & (o) & 1.5 & 100783 & (e) & 0.5 & -2.69 & $1.54 \mathrm{E}+06$ & $2.16 \mathrm{E}-03$ & -3.655 \\
\hline 2967.410 & 49352 & (o) & 2.5 & 83041 & (e) & 3.5 & -0.86 & $1.04 \mathrm{E}+08$ & $2.60 \mathrm{E}-02$ & -1.383 \\
\hline 2967.711 & 49006 & (o) & 1.5 & 82692 & (e) & 0.5 & -1.12 & $5.68 \mathrm{E}+07$ & $5.68 \mathrm{E}-02$ & -1.01 \\
\hline 2967.777 & 66355 & (o) & 1.5 & 100040 & (e) & 1.5 & -0.85 & $1.09 \mathrm{E}+08$ & $7.36 \mathrm{E}-02$ & -1.087 \\
\hline 2971.927 & 67012 & (o) & 2.5 & 100650 & (e) & 2.5 & -1.50 & $2.36 \mathrm{E}+07$ & $1.06 \mathrm{E}-02$ & -1.379 \\
\hline 2972.379 & 67860 & (o) & 0.5 & 101493 & (e) & 1.5 & -2.76 & $1.31 \mathrm{E}+06$ & $9.17 \mathrm{E}-04$ & -2.831 \\
\hline 2973.107 & 67868 & (o) & 2.5 & 101493 & (e) & 1.5 & -1.81 & $1.17 \mathrm{E}+07$ & $8.19 \mathrm{E}-03$ & -1.7 \\
\hline 2973.321 & 67870 & (o) & 1.5 & 101493 & (e) & 1.5 & -1.31 & $3.74 \mathrm{E}+07$ & $2.62 \mathrm{E}-02$ & -1.623 \\
\hline 2977.093 & 67070 & (o) & 1.5 & 100650 & (e) & 2.5 & -1.82 & $1.15 \mathrm{E}+07$ & $5.18 \mathrm{E}-03$ & -3.143 \\
\hline 2981.590 & 49352 & (o) & 2.5 & 82881 & (e) & 2.5 & -1.07 & $6.41 \mathrm{E}+07$ & $2.14 \mathrm{E}-02$ & -1.056 \\
\hline 2988.305 & 67868 & (o) & 2.5 & 101322 & (e) & 3.5 & -0.23 & $4.37 \mathrm{E}+08$ & $1.47 \mathrm{E}-01$ & -0.27 \\
\hline 2991.222 & 66256 & (o) & 0.5 & 99678 & (e) & 0.5 & -1.46 & $2.62 \mathrm{E}+07$ & $3.54 \mathrm{E}-02$ & -1.441 \\
\hline 2992.070 & 49352 & (o) & 2.5 & 82764 & (e) & 1.5 & -0.76 & $1.29 \mathrm{E}+08$ & $6.45 \mathrm{E}-02$ & -0.842 \\
\hline 2992.354 & 67868 & (o) & 2.5 & 101277 & (e) & 2.5 & -0.79 & $1.21 \mathrm{E}+08$ & $5.45 \mathrm{E}-02$ & -0.652 \\
\hline 2992.571 & 67870 & (o) & 1.5 & 101277 & (e) & 2.5 & -3.17 & $5.09 \mathrm{E}+05$ & $2.29 \mathrm{E}-04$ & -0.466 \\
\hline 2992.823 & 67379 & (o) & 1.5 & 100783 & (e) & 0.5 & -1.63 & $1.75 \mathrm{E}+07$ & $2.45 \mathrm{E}-02$ & -1.621 \\
\hline 2993.034 & 67875 & (o) & 3.5 & 101277 & (e) & 2.5 & -2.18 & $4.86 \mathrm{E}+06$ & $2.19 \mathrm{E}-03$ & -1.648 \\
\hline 2993.966 & 66649 & (o) & 1.5 & 100040 & (e) & 1.5 & -3.85 & $1.05 \mathrm{E}+05$ & $7.09 \mathrm{E}-05$ & -1.851 \\
\hline 2994.444 & 67860 & (o) & 0.5 & 101245 & (e) & 1.5 & -0.57 & $1.99 \mathrm{E}+08$ & $1.34 \mathrm{E}-01$ & -0.56 \\
\hline 2995.182 & 67868 & (o) & 2.5 & 101245 & (e) & 1.5 & -2.09 & $6.01 \mathrm{E}+06$ & $4.06 \mathrm{E}-03$ & -1.733 \\
\hline 2995.400 & 67870 & (o) & 1.5 & 101245 & (e) & 1.5 & -3.01 & $7.35 \mathrm{E}+05$ & $4.96 \mathrm{E}-04$ & -0.889 \\
\hline 2998.970 & 49706 & (o) & 2.5 & 83041 & (e) & 3.5 & -0.69 & $1.52 \mathrm{E}+08$ & $3.80 \mathrm{E}-02$ & -0.676 \\
\hline 3000.047 & 66355 & (o) & 1.5 & 99678 & (e) & 0.5 & -0.53 & $2.19 \mathrm{E}+08$ & $2.96 \mathrm{E}-01$ & -0.617 \\
\hline 3000.625 & 67334 & (o) & 3.5 & 100650 & (e) & 2.5 & -3.38 & $3.13 \mathrm{E}+05$ & $1.41 \mathrm{E}-04$ & -3.282 \\
\hline 3000.632 & 49565 & (o) & 1.5 & 82881 & (e) & 2.5 & -0.61 & $1.82 \mathrm{E}+08$ & $6.07 \mathrm{E}-02$ & -0.656 \\
\hline
\end{tabular}


Table 4 (continued)

\begin{tabular}{|c|c|c|c|c|c|c|c|c|c|c|}
\hline $\begin{array}{c}\text { Wavelength } \\
(\AA)\end{array}$ & $\begin{array}{c}E \text { (lower) } \\
\left(\mathrm{cm}^{-1}\right)\end{array}$ & Parity (lower) & $J$ (lower) & $\begin{array}{c}E \text { (upper) } \\
\left(\mathrm{cm}^{-1}\right)\end{array}$ & Parity (upper) & $J$ (upper) & $\log (g f)(\mathrm{HFR}+\mathrm{CPOL})$ & $\begin{array}{c}g A(\mathrm{HFR}+\mathrm{CPOL}) \\
\left(\mathrm{s}^{-1}\right)\end{array}$ & $B F(\mathrm{HFR}+\mathrm{CPOL})$ & $\begin{array}{c}\log (g f) \\
\text { (Kurucz) }\end{array}$ \\
\hline 3000.921 & 66727 & (o) & 2.5 & 100040 & (e) & 1.5 & -0.59 & $1.91 \mathrm{E}+08$ & $1.29 \mathrm{E}-01$ & -0.579 \\
\hline 3001.546 & 67344 & (o) & 2.5 & 100650 & (e) & 2.5 & -2.35 & $3.33 \mathrm{E}+06$ & $1.50 \mathrm{E}-03$ & -4.481 \\
\hline 3004.730 & 67379 & (o) & 1.5 & 100650 & (e) & 2.5 & -2.44 & $2.71 \mathrm{E}+06$ & $1.22 \mathrm{E}-03$ & -3.257 \\
\hline 3004.749 & 49493 & (o) & 0.5 & 82764 & (e) & 1.5 & -0.78 & $1.22 \mathrm{E}+08$ & $6.10 \mathrm{E}-02$ & -0.826 \\
\hline 3005.434 & 67387 & (o) & 2.5 & 100650 & (e) & 2.5 & -2.05 & $6.66 \mathrm{E}+06$ & $3.00 \mathrm{E}-03$ & -2.256 \\
\hline 3006.009 & 67393 & (o) & 3.5 & 100650 & (e) & 2.5 & -2.50 & $2.33 \mathrm{E}+06$ & $1.05 E-03$ & -1.874 \\
\hline 3007.973 & 49646 & (o) & 3.5 & 82881 & (e) & 2.5 & -0.45 & $2.63 \mathrm{E}+08$ & $8.77 \mathrm{E}-02$ & -0.43 \\
\hline 3011.234 & 49493 & (o) & 0.5 & 82692 & (e) & 0.5 & -1.33 & $3.47 \mathrm{E}+07$ & $3.47 \mathrm{E}-02$ & -1.353 \\
\hline 3011.247 & 49565 & (o) & 1.5 & 82764 & (e) & 1.5 & -2.51 & $2.27 \mathrm{E}+06$ & $1.14 \mathrm{E}-03$ & -2.453 \\
\hline 3012.326 & 68306 & (o) & 1.5 & 101493 & (e) & 1.5 & -2.01 & $7.27 \mathrm{E}+06$ & $5.09 \mathrm{E}-03$ & -1.57 \\
\hline 3013.455 & 49706 & (o) & 2.5 & 82881 & (e) & 2.5 & -1.21 & $4.56 \mathrm{E}+07$ & $1.52 \mathrm{E}-02$ & -1.1 \\
\hline 3014.045 & 66872 & (o) & 0.5 & 100040 & (e) & 1.5 & -3.15 & $5.19 \mathrm{E}+05$ & $3.50 \mathrm{E}-04$ & \\
\hline 3017.760 & 49565 & (o) & 1.5 & 82692 & (e) & 0.5 & -0.83 & $1.08 \mathrm{E}+08$ & $1.08 \mathrm{E}-01$ & -0.817 \\
\hline 3024.160 & 49706 & (o) & 2.5 & 82764 & (e) & 1.5 & -0.79 & $1.19 \mathrm{E}+08$ & $5.95 \mathrm{E}-02$ & -0.672 \\
\hline 3024.217 & 67012 & (o) & 2.5 & 100069 & (e) & 3.5 & -1.24 & $4.14 \mathrm{E}+07$ & $1.45 \mathrm{E}-02$ & -2.615 \\
\hline 3026.811 & 66649 & (o) & 1.5 & 99678 & (e) & 0.5 & -3.21 & $4.43 \mathrm{E}+05$ & $5.98 \mathrm{E}-04$ & -1.684 \\
\hline 3026.844 & 67012 & (o) & 2.5 & 100040 & (e) & 1.5 & -3.95 & $8.04 \mathrm{E}+04$ & $5.43 \mathrm{E}-05$ & -3.786 \\
\hline 3032.086 & 68306 & (o) & 1.5 & 101277 & (e) & 2.5 & -0.39 & $3.04 \mathrm{E}+08$ & $1.37 \mathrm{E}-01$ & -1.307 \\
\hline 3032.202 & 67070 & (o) & 1.5 & 100040 & (e) & 1.5 & -1.65 & $1.64 \mathrm{E}+07$ & $1.11 \mathrm{E}-02$ & -1.619 \\
\hline 3034.990 & 68306 & (o) & 1.5 & 101245 & (e) & 1.5 & -0.93 & $8.69 \mathrm{E}+07$ & $5.87 \mathrm{E}-02$ & -1.661 \\
\hline 3036.482 & 67860 & (o) & 0.5 & 100783 & (e) & 0.5 & -2.64 & $1.63 \mathrm{E}+06$ & $2.28 \mathrm{E}-03$ & -2.655 \\
\hline 3037.465 & 67870 & (o) & 1.5 & 100783 & (e) & 0.5 & -1.93 & $8.47 \mathrm{E}+06$ & $1.19 \mathrm{E}-02$ & -3.727 \\
\hline 3037.566 & 51584 & (o) & 1.5 & 84496 & (e) & 2.5 & -2.02 & $6.94 \mathrm{E}+06$ & $3.35 E-03$ & -2.068 \\
\hline 3037.752 & 68583 & (o) & 2.5 & 101493 & (e) & 1.5 & -1.60 & $1.81 \mathrm{E}+07$ & $1.27 \mathrm{E}-02$ & -1.991 \\
\hline 3045.460 & 51669 & (o) & 2.5 & 84496 & (e) & 2.5 & -0.69 & $1.50 \mathrm{E}+08$ & $7.25 \mathrm{E}-02$ & -0.725 \\
\hline 3047.335 & 66872 & (o) & 0.5 & 99678 & (e) & 0.5 & -2.56 & $1.98 \mathrm{E}+06$ & $2.67 \mathrm{E}-03$ & -2.778 \\
\hline 3049.505 & 67868 & (o) & 2.5 & 100650 & (e) & 2.5 & -1.25 & $4.02 \mathrm{E}+07$ & $1.81 \mathrm{E}-02$ & -1.421 \\
\hline 3049.731 & 67870 & (o) & 1.5 & 100650 & (e) & 2.5 & -0.39 & $2.89 \mathrm{E}+08$ & $1.30 \mathrm{E}-01$ & -1.679 \\
\hline 3050.211 & 67875 & (o) & 3.5 & 100650 & (e) & 2.5 & -0.66 & $1.56 \mathrm{E}+08$ & $7.02 \mathrm{E}-02$ & -0.708 \\
\hline 3053.620 & 68583 & (o) & 2.5 & 101322 & (e) & 3.5 & -1.83 & $1.06 \mathrm{E}+07$ & $3.58 \mathrm{E}-03$ & -1.797 \\
\hline 3053.849 & 51584 & (o) & 1.5 & 84320 & (e) & 1.5 & -0.78 & $1.20 \mathrm{E}+08$ & $8.70 \mathrm{E}-02$ & -0.813 \\
\hline 3054.893 & 67344 & (o) & 2.5 & 100069 & (e) & 3.5 & -0.19 & $4.66 \mathrm{E}+08$ & $1.63 \mathrm{E}-01$ & -0.118 \\
\hline 3056.580 & 51789 & (o) & 3.5 & 84496 & (e) & 2.5 & 0.05 & $8.06 \mathrm{E}+08$ & $3.90 \mathrm{E}-01$ & 0.018 \\
\hline 3057.848 & 68583 & (o) & 2.5 & 101277 & (e) & 2.5 & -2.91 & $8.65 E+05$ & $3.89 \mathrm{E}-04$ & -2.654 \\
\hline 3058.921 & 67387 & (o) & 2.5 & 100069 & (e) & 3.5 & -1.91 & $8.78 \mathrm{E}+06$ & $3.07 \mathrm{E}-03$ & -2.438 \\
\hline 3060.801 & 68583 & (o) & 2.5 & 101245 & (e) & 1.5 & -3.06 & $6.14 \mathrm{E}+05$ & $4.14 \mathrm{E}-04$ & -3.385 \\
\hline 3060.877 & 67379 & (o) & 1.5 & 100040 & (e) & 1.5 & -2.15 & $5.06 \mathrm{E}+06$ & $3.42 \mathrm{E}-03$ & -2.491 \\
\hline 3061.608 & 67387 & (o) & 2.5 & 100040 & (e) & 1.5 & -3.90 & $9.04 \mathrm{E}+04$ & $6.10 \mathrm{E}-05$ & -3.323 \\
\hline 3061.828 & 51669 & (o) & 2.5 & 84320 & (e) & 1.5 & -0.13 & $5.31 \mathrm{E}+08$ & $3.85 \mathrm{E}-01$ & -0.162 \\
\hline 3064.179 & 51584 & (o) & 1.5 & 84210 & (e) & 0.5 & -0.34 & $3.28 \mathrm{E}+08$ & $4.76 \mathrm{E}-01$ & -0.37 \\
\hline 3065.896 & 67070 & (o) & 1.5 & 99678 & (e) & 0.5 & -2.81 & $1.09 \mathrm{E}+06$ & $1.47 \mathrm{E}-03$ & -2.554 \\
\hline 3074.445 & 68760 & (o) & 3.5 & 101277 & (e) & 2.5 & -2.76 & $1.22 \mathrm{E}+06$ & $5.49 \mathrm{E}-04$ & -3.059 \\
\hline 3078.183 & 68306 & (0) & 1.5 & 100783 & (e) & 0.5 & -2.79 & $1.16 \mathrm{E}+06$ & $1.62 \mathrm{E}-03$ & -1.831 \\
\hline 3082.358 & 68843 & (o) & 3.5 & 101277 & (e) & 2.5 & -3.06 & $6.10 \mathrm{E}+05$ & $2.75 \mathrm{E}-04$ & -2.627 \\
\hline 3090.780 & 68306 & (o) & 1.5 & 100650 & (e) & 2.5 & -2.44 & $2.58 \mathrm{E}+06$ & $1.16 \mathrm{E}-03$ & -0.482 \\
\hline 3095.215 & 67379 & (o) & 1.5 & 99678 & (e) & 0.5 & -3.54 & $2.00 \mathrm{E}+05$ & $2.70 \mathrm{E}-04$ & -3.415 \\
\hline 3104.586 & 67868 & (o) & 2.5 & 100069 & (e) & 3.5 & -3.92 & $8.11 \mathrm{E}+04$ & $2.84 \mathrm{E}-05$ & -3.21 \\
\hline 3106.560 & 67860 & (o) & 0.5 & 100040 & (e) & 1.5 & -2.16 & $4.69 \mathrm{E}+06$ & $3.17 \mathrm{E}-03$ & -2.265 \\
\hline 3107.354 & 67868 & (o) & 2.5 & 100040 & (e) & 1.5 & -1.18 & $4.53 \mathrm{E}+07$ & $3.06 \mathrm{E}-02$ & -1.182 \\
\hline 3107.589 & 67870 & (o) & 1.5 & 100040 & (e) & 1.5 & -0.84 & $9.88 \mathrm{E}+07$ & $6.67 \mathrm{E}-02$ & -0.999 \\
\hline 3110.033 & 69348 & (o) & 1.5 & 101493 & (e) & 1.5 & -1.00 & $6.92 \mathrm{E}+07$ & $4.84 \mathrm{E}-02$ & -1.01 \\
\hline 3117.553 & 68583 & (0) & 2.5 & 100650 & (e) & 2.5 & -2.67 & $1.46 \mathrm{E}+06$ & $6.57 \mathrm{E}-04$ & -2.759 \\
\hline 3122.638 & 69478 & (o) & 2.5 & 101493 & (e) & 1.5 & -0.23 & $4.02 \mathrm{E}+08$ & $2.81 \mathrm{E}-01$ & -0.204 \\
\hline 3131.100 & 69348 & (o) & 1.5 & 101277 & (e) & 2.5 & -2.81 & $1.08 \mathrm{E}+06$ & $4.86 \mathrm{E}-04$ & -2.617 \\
\hline 3134.197 & 69348 & (o) & 1.5 & 101245 & (e) & 1.5 & -2.39 & $2.80 \mathrm{E}+06$ & $1.89 \mathrm{E}-03$ & -2.725 \\
\hline 3138.391 & 69639 & (o) & 1.5 & 101493 & (e) & 1.5 & -2.31 & $3.33 \mathrm{E}+06$ & $2.33 \mathrm{E}-03$ & -2.389 \\
\hline 3139.408 & 69478 & (o) & 2.5 & 101322 & (e) & 3.5 & -3.08 & $5.60 \mathrm{E}+05$ & $1.89 \mathrm{E}-04$ & -2.51 \\
\hline 3141.936 & 67860 & (o) & 0.5 & 99678 & (e) & 0.5 & -1.60 & $1.70 \mathrm{E}+07$ & $2.30 \mathrm{E}-02$ & -1.619 \\
\hline 3142.988 & 67870 & (o) & 1.5 & 99678 & (e) & 0.5 & -1.24 & $3.92 \mathrm{E}+07$ & $5.29 \mathrm{E}-02$ & -2.673 \\
\hline 3143.033 & 68843 & (o) & 3.5 & 100650 & (e) & 2.5 & -2.17 & $4.54 \mathrm{E}+06$ & $2.04 \mathrm{E}-03$ & \\
\hline 3143.877 & 69478 & (o) & 2.5 & 101277 & (e) & 2.5 & -2.50 & $2.14 \mathrm{E}+06$ & $9.63 \mathrm{E}-04$ & -2.989 \\
\hline 3146.664 & 69506 & (o) & 3.5 & 101277 & (e) & 2.5 & -1.62 & $1.60 \mathrm{E}+07$ & $7.20 \mathrm{E}-03$ & -1.652 \\
\hline 3146.999 & 69478 & (o) & 2.5 & 101245 & (e) & 1.5 & -2.35 & $2.97 \mathrm{E}+06$ & $2.00 \mathrm{E}-03$ & -2.566 \\
\hline 3150.221 & 68306 & (o) & 1.5 & 100040 & (e) & 1.5 & -1.34 & $3.10 \mathrm{E}+07$ & $2.09 \mathrm{E}-02$ & -0.973 \\
\hline 3159.846 & 69639 & (o) & 1.5 & 101277 & (e) & 2.5 & -2.97 & $7.16 \mathrm{E}+05$ & $3.22 \mathrm{E}-04$ & -2.89 \\
\hline 3162.999 & 69639 & (o) & 1.5 & 101245 & (e) & 1.5 & -2.10 & $5.21 \mathrm{E}+06$ & $3.52 \mathrm{E}-03$ & -2.39 \\
\hline
\end{tabular}


Table 4 (continued)

\begin{tabular}{|c|c|c|c|c|c|c|c|c|c|c|}
\hline $\begin{array}{c}\text { Wavelength } \\
(\AA)\end{array}$ & $\begin{array}{c}E \text { (lower) } \\
\left(\mathrm{cm}^{-1}\right)\end{array}$ & Parity (lower) & $J$ (lower) & $\begin{array}{c}E \text { (upper) } \\
\left(\mathrm{cm}^{-1}\right)\end{array}$ & Parity (upper) & $J$ (upper) & $\log (g f)(\mathrm{HFR}+\mathrm{CPOL})$ & $\begin{array}{c}g A(\mathrm{HFR}+\mathrm{CPOL}) \\
\left(\mathrm{s}^{-1}\right)\end{array}$ & $B F(\mathrm{HFR}+\mathrm{CPOL})$ & $\begin{array}{c}\log (g f) \\
\text { (Kurucz) }\end{array}$ \\
\hline 3169.784 & 69954 & (o) & 2.5 & 101493 & (e) & 1.5 & -0.78 & $1.11 \mathrm{E}+08$ & $7.77 \mathrm{E}-02$ & -0.853 \\
\hline 3175.142 & 68583 & (o) & 2.5 & 100069 & (e) & 3.5 & -1.98 & $6.82 \mathrm{E}+06$ & $2.39 \mathrm{E}-03$ & -1.919 \\
\hline 3178.038 & 68583 & (o) & 2.5 & 100040 & (e) & 1.5 & -2.58 & $1.71 \mathrm{E}+06$ & $1.15 \mathrm{E}-03$ & -2.579 \\
\hline 3180.280 & 69348 & (o) & 1.5 & 100783 & (e) & 0.5 & -0.44 & $2.42 \mathrm{E}+08$ & $3.39 \mathrm{E}-01$ & -0.417 \\
\hline 3186.526 & 69903 & (0) & 3.5 & 101277 & (e) & 2.5 & -2.81 & $1.01 \mathrm{E}+06$ & $4.55 \mathrm{E}-04$ & -3.448 \\
\hline 3186.604 & 68306 & (o) & 1.5 & 99678 & (e) & 0.5 & -1.69 & $1.37 \mathrm{E}+07$ & $1.85 \mathrm{E}-02$ & -1.004 \\
\hline 3186.643 & 51669 & (o) & 2.5 & 83041 & (e) & 3.5 & -3.10 & $5.31 \mathrm{E}+05$ & $1.33 \mathrm{E}-04$ & -3.105 \\
\hline 3187.065 & 69954 & (o) & 2.5 & 101322 & (e) & 3.5 & -3.09 & $5.37 \mathrm{E}+05$ & $1.81 \mathrm{E}-04$ & -2.9 \\
\hline 3191.671 & 69954 & (o) & 2.5 & 101277 & (e) & 2.5 & -2.29 & $3.39 \mathrm{E}+06$ & $1.53 \mathrm{E}-03$ & -2.698 \\
\hline 3193.729 & 69348 & (o) & 1.5 & 100650 & (e) & 2.5 & -3.25 & $3.71 \mathrm{E}+05$ & $1.67 \mathrm{E}-04$ & -3.228 \\
\hline 3194.271 & 51584 & (o) & 1.5 & 82881 & (e) & 2.5 & -3.58 & $1.73 \mathrm{E}+05$ & $5.77 \mathrm{E}-05$ & -3.557 \\
\hline 3194.888 & 69954 & (o) & 2.5 & 101245 & (e) & 1.5 & -2.04 & $6.04 \mathrm{E}+06$ & $4.08 \mathrm{E}-03$ & -2.025 \\
\hline 3203.002 & 51669 & (o) & 2.5 & 82881 & (e) & 2.5 & -2.67 & $1.41 \mathrm{E}+06$ & $4.70 \mathrm{E}-04$ & -2.59 \\
\hline 3206.302 & 51584 & (o) & 1.5 & 82764 & (e) & 1.5 & -3.27 & $3.52 \mathrm{E}+05$ & $1.76 \mathrm{E}-04$ & -3.176 \\
\hline 3206.669 & 70317 & (o) & 2.5 & 101493 & (e) & 1.5 & -3.39 & $2.62 \mathrm{E}+05$ & $1.83 \mathrm{E}-04$ & \\
\hline 3207.023 & 69478 & (o) & 2.5 & 100650 & (e) & 2.5 & -2.62 & $1.54 \mathrm{E}+06$ & $6.93 \mathrm{E}-04$ & -2.403 \\
\hline 3209.924 & 69506 & (o) & 3.5 & 100650 & (e) & 2.5 & -2.95 & $7.10 \mathrm{E}+05$ & $3.20 \mathrm{E}-04$ & -3.28 \\
\hline 3209.940 & 69639 & (o) & 1.5 & 100783 & (e) & 0.5 & -1.68 & $1.33 \mathrm{E}+07$ & $1.86 \mathrm{E}-02$ & -1.76 \\
\hline 3213.688 & 51584 & (o) & 1.5 & 82692 & (e) & 0.5 & -3.86 & $8.92 \mathrm{E}+04$ & $8.92 \mathrm{E}-05$ & -3.71 \\
\hline 3213.784 & 73436 & (o) & 2.5 & 104543 & (e) & 3.5 & -3.53 & $1.85 \mathrm{E}+05$ & $5.32 \mathrm{E}-05$ & -3.277 \\
\hline 3215.099 & 51669 & (o) & 2.5 & 82764 & (e) & 1.5 & -3.35 & $2.93 \mathrm{E}+05$ & $1.47 \mathrm{E}-04$ & -3.162 \\
\hline 3215.303 & 51789 & (o) & 3.5 & 82881 & (e) & 2.5 & -3.21 & $3.99 \mathrm{E}+05$ & $1.33 \mathrm{E}-04$ & -2.993 \\
\hline 3224.356 & 70317 & (o) & 2.5 & 101322 & (e) & 3.5 & -3.22 & $3.91 \mathrm{E}+05$ & $1.32 \mathrm{E}-04$ & -3.506 \\
\hline 3229.071 & 70317 & (o) & 2.5 & 101277 & (e) & 2.5 & -2.21 & $3.92 \mathrm{E}+06$ & $1.76 \mathrm{E}-03$ & -2.359 \\
\hline 3232.364 & 70317 & (o) & 2.5 & 101245 & (e) & 1.5 & -1.36 & $2.81 \mathrm{E}+07$ & $1.90 \mathrm{E}-02$ & -1.296 \\
\hline 3234.430 & 70584 & (o) & 2.5 & 101493 & (e) & 1.5 & -2.86 & $8.74 \mathrm{E}+05$ & $6.12 \mathrm{E}-04$ & -3.448 \\
\hline 3240.603 & 70427 & (o) & 3.5 & 101277 & (e) & 2.5 & -1.18 & $4.17 \mathrm{E}+07$ & $1.88 \mathrm{E}-02$ & -1.122 \\
\hline 3252.425 & 70584 & (o) & 2.5 & 101322 & (e) & 3.5 & -3.94 & $7.24 \mathrm{E}+04$ & $2.44 \mathrm{E}-05$ & -3.299 \\
\hline 3256.771 & 69954 & (o) & 2.5 & 100650 & (e) & 2.5 & -2.71 & $1.23 \mathrm{E}+06$ & $5.54 \mathrm{E}-04$ & -2.766 \\
\hline 3257.222 & 70584 & (o) & 2.5 & 101277 & (e) & 2.5 & -3.48 & $2.10 \mathrm{E}+05$ & $9.45 \mathrm{E}-05$ & \\
\hline 3257.236 & 69348 & (o) & 1.5 & 100040 & (e) & 1.5 & -2.50 & $2.01 \mathrm{E}+06$ & $1.36 \mathrm{E}-03$ & -2.332 \\
\hline 3260.573 & 70584 & (o) & 2.5 & 101245 & (e) & 1.5 & -2.37 & $2.72 \mathrm{E}+06$ & $1.84 \mathrm{E}-03$ & -3.826 \\
\hline 3267.998 & 69478 & (0) & 2.5 & 100069 & (e) & 3.5 & -2.47 & $2.12 \mathrm{E}+06$ & $7.42 \mathrm{E}-04$ & -2.519 \\
\hline 3271.065 & 69478 & (0) & 2.5 & 100040 & (e) & 1.5 & -2.17 & $4.22 \mathrm{E}+06$ & $2.85 \mathrm{E}-03$ & -1.948 \\
\hline 3285.891 & 70852 & (o) & 3.5 & 101277 & (e) & 2.5 & -3.25 & $3.52 \mathrm{E}+05$ & $1.58 \mathrm{E}-04$ & -4.186 \\
\hline 3288.356 & 69639 & (o) & 1.5 & 100040 & (e) & 1.5 & -3.17 & $4.16 \mathrm{E}+05$ & $2.81 \mathrm{E}-04$ & -3.257 \\
\hline 3296.148 & 69348 & (o) & 1.5 & 99678 & (e) & 0.5 & -2.19 & $4.01 \mathrm{E}+06$ & $5.41 \mathrm{E}-03$ & -2.078 \\
\hline 3307.647 & 74319 & (o) & 2.5 & 104543 & (e) & 3.5 & -2.80 & $9.57 \mathrm{E}+05$ & $2.75 \mathrm{E}-04$ & -2.323 \\
\hline 3319.671 & 69954 & (o) & 2.5 & 100069 & (e) & 3.5 & -2.45 & $2.18 \mathrm{E}+06$ & $7.63 \mathrm{E}-04$ & -2.225 \\
\hline 3320.531 & 74436 & (o) & 2.5 & 104543 & (e) & 3.5 & -2.55 & $1.69 \mathrm{E}+06$ & $4.86 \mathrm{E}-04$ & -1.798 \\
\hline 3322.836 & 69954 & (o) & 2.5 & 100040 & (e) & 1.5 & -2.28 & $3.18 \mathrm{E}+06$ & $2.15 \mathrm{E}-03$ & -2.308 \\
\hline 3325.811 & 74484 & (0) & 2.5 & 104543 & (e) & 3.5 & -1.06 & $5.06 \mathrm{E}+07$ & $1.45 \mathrm{E}-02$ & -2.715 \\
\hline 3328.020 & 69639 & (0) & 1.5 & 99678 & (e) & 0.5 & -3.03 & $5.58 \mathrm{E}+05$ & $7.53 \mathrm{E}-04$ & -3.11 \\
\hline 3332.806 & 54499 & (o) & 1.5 & 84496 & (e) & 2.5 & -0.60 & $1.53 \mathrm{E}+08$ & $7.40 \mathrm{E}-02$ & -0.615 \\
\hline 3338.074 & 74718 & (o) & 1.5 & 104666 & (e) & 0.5 & -3.43 & $2.25 \mathrm{E}+05$ & $2.93 \mathrm{E}-04$ & \\
\hline 3343.276 & 54418 & (o) & 0.5 & 84320 & (e) & 1.5 & -0.76 & $1.04 \mathrm{E}+08$ & $7.54 \mathrm{E}-02$ & -0.781 \\
\hline 3346.876 & 54626 & (o) & 2.5 & 84496 & (e) & 2.5 & -0.23 & $3.54 \mathrm{E}+08$ & $1.71 \mathrm{E}-01$ & -0.246 \\
\hline 3352.418 & 54499 & (o) & 1.5 & 84320 & (e) & 1.5 & -0.57 & $1.61 \mathrm{E}+08$ & $1.17 \mathrm{E}-01$ & -0.586 \\
\hline 3353.336 & 74854 & (o) & 0.5 & 104666 & (e) & 0.5 & -3.38 & $2.49 \mathrm{E}+05$ & $3.24 \mathrm{E}-04$ & \\
\hline 3355.661 & 54418 & (o) & 0.5 & 84210 & (e) & 0.5 & -0.78 & $9.82 \mathrm{E}+07$ & $1.42 \mathrm{E}-01$ & -0.802 \\
\hline 3360.149 & 70317 & (0) & 2.5 & 100069 & (e) & 3.5 & -0.85 & $8.22 \mathrm{E}+07$ & $2.88 \mathrm{E}-02$ & -0.941 \\
\hline 3360.837 & 74920 & (0) & 0.5 & 104666 & (e) & 0.5 & -2.75 & $1.01 \mathrm{E}+06$ & $1.31 \mathrm{E}-03$ & \\
\hline 3364.772 & 54784 & (o) & 3.5 & 84496 & (e) & 2.5 & -0.78 & $9.71 \mathrm{E}+07$ & $4.69 \mathrm{E}-02$ & -0.817 \\
\hline 3364.870 & 54499 & (o) & 1.5 & 84210 & (e) & 0.5 & -0.82 & $9.03 \mathrm{E}+07$ & $1.31 \mathrm{E}-01$ & -0.841 \\
\hline 3366.655 & 54626 & (o) & 2.5 & 84320 & (e) & 1.5 & -0.68 & $1.22 \mathrm{E}+08$ & $8.85 \mathrm{E}-02$ & -0.713 \\
\hline 3368.118 & 74985 & (o) & 1.5 & 104666 & (e) & 0.5 & -2.75 & $1.02 \mathrm{E}+06$ & $1.33 \mathrm{E}-03$ & \\
\hline 3390.643 & 70584 & (o) & 2.5 & 100069 & (e) & 3.5 & -2.08 & $4.84 \mathrm{E}+06$ & $1.69 \mathrm{E}-03$ & -3.89 \\
\hline 3518.172 & 54626 & (o) & 2.5 & 83041 & (e) & 3.5 & -3.27 & $2.88 \mathrm{E}+05$ & $7.20 \mathrm{E}-05$ & -3.109 \\
\hline 3522.401 & 54499 & (o) & 1.5 & 82881 & (e) & 2.5 & -3.55 & $1.50 \mathrm{E}+05$ & $5.00 \mathrm{E}-05$ & -3.496 \\
\hline 3538.122 & 54626 & (0) & 2.5 & 82881 & (e) & 2.5 & -3.80 & $8.40 \mathrm{E}+04$ & $2.80 \mathrm{E}-05$ & -4.13 \\
\hline 3546.027 & 54499 & (o) & 1.5 & 82692 & (e) & 0.5 & -3.73 & $9.82 \mathrm{E}+04$ & $9.82 \mathrm{E}-05$ & -3.599 \\
\hline 3552.889 & 54626 & (o) & 2.5 & 82764 & (e) & 1.5 & -3.34 & $2.40 \mathrm{E}+05$ & $1.20 \mathrm{E}-04$ & -3.245 \\
\hline 3558.127 & 54784 & (o) & 3.5 & 82881 & (e) & 2.5 & -3.36 & $2.29 \mathrm{E}+05$ & $7.63 \mathrm{E}-05$ & -3.284 \\
\hline 3585.046 & 73436 & (o) & 2.5 & 101322 & (e) & 3.5 & -2.91 & $6.24 \mathrm{E}+05$ & $2.11 \mathrm{E}-04$ & -4.289 \\
\hline 3591.173 & 73407 & (o) & 0.5 & 101245 & (e) & 1.5 & -3.43 & $1.89 \mathrm{E}+05$ & $1.28 \mathrm{E}-04$ & \\
\hline 3591.817 & 73412 & (o) & 1.5 & 101245 & (e) & 1.5 & -3.56 & $1.40 \mathrm{E}+05$ & $9.45 \mathrm{E}-05$ & \\
\hline
\end{tabular}


Table 4 (continued)

\begin{tabular}{|c|c|c|c|c|c|c|c|c|c|c|}
\hline $\begin{array}{c}\text { Wavelength } \\
(\AA)\end{array}$ & $\begin{array}{c}\text { E(lower) } \\
\left(\mathrm{cm}^{-1}\right)\end{array}$ & Parity (lower) & $J$ (lower) & $\begin{array}{c}E \text { (upper) } \\
\left(\mathrm{cm}^{-1}\right)\end{array}$ & Parity (upper) & $J$ (upper) & $\log (g f)(\mathrm{HFR}+\mathrm{CPOL})$ & $\begin{array}{c}g A(\mathrm{HFR}+\mathrm{CPOL}) \\
\left(\mathrm{s}^{-1}\right)\end{array}$ & $B F(\mathrm{HFR}+\mathrm{CPOL})$ & $\begin{array}{c}\log (g f) \\
\text { (Kurucz) }\end{array}$ \\
\hline 3628.010 & 76988 & (o) & 2.5 & 104543 & (e) & 3.5 & -1.34 & $2.28 \mathrm{E}+07$ & $6.56 \mathrm{E}-03$ & -1.424 \\
\hline 3670.219 & 73412 & (o) & 1.5 & 100650 & (e) & 2.5 & -3.59 & $1.23 \mathrm{E}+05$ & $5.54 \mathrm{E}-05$ & -3.547 \\
\hline 3673.489 & 73436 & (o) & 2.5 & 100650 & (e) & 2.5 & -2.86 & $6.61 \mathrm{E}+05$ & $2.97 \mathrm{E}-04$ & -2.699 \\
\hline 3680.180 & 73486 & (o) & 3.5 & 100650 & (e) & 2.5 & -2.87 & $6.48 \mathrm{E}+05$ & $2.92 \mathrm{E}-04$ & -2.619 \\
\hline 3701.428 & 74484 & (o) & 2.5 & 101493 & (e) & 1.5 & -3.34 & $2.17 \mathrm{E}+05$ & $1.52 \mathrm{E}-04$ & -4.223 \\
\hline 3706.540 & 74273 & (o) & 1.5 & 101245 & (e) & 1.5 & -3.48 & $1.57 \mathrm{E}+05$ & $1.06 \mathrm{E}-04$ & -4.124 \\
\hline 3709.098 & 77713 & (o) & 1.5 & 104666 & (e) & 0.5 & -3.07 & $4.05 \mathrm{E}+05$ & $5.27 \mathrm{E}-04$ & \\
\hline 3717.930 & 77777 & (o) & 0.5 & 104666 & (e) & 0.5 & -1.78 & $7.71 \mathrm{E}+06$ & $1.00 \mathrm{E}-02$ & \\
\hline 3733.729 & 74718 & (o) & 1.5 & 101493 & (e) & 1.5 & -3.52 & $1.44 \mathrm{E}+05$ & $1.01 \mathrm{E}-04$ & -3.584 \\
\hline 3752.833 & 74854 & (o) & 0.5 & 101493 & (e) & 1.5 & -3.88 & $6.29 \mathrm{E}+04$ & $4.40 \mathrm{E}-05$ & -4.396 \\
\hline 3753.634 & 73407 & (o) & 0.5 & 100040 & (e) & 1.5 & -3.88 & $6.00 \mathrm{E}+04$ & $4.05 \mathrm{E}-05$ & -3.767 \\
\hline 3754.338 & 73412 & (o) & 1.5 & 100040 & (e) & 1.5 & -3.32 & $2.18 \mathrm{E}+05$ & $1.47 \mathrm{E}-04$ & -3.141 \\
\hline 3757.213 & 77935 & (o) & 2.5 & 104543 & (e) & 3.5 & -3.42 & $1.78 \mathrm{E}+05$ & $5.12 \mathrm{E}-05$ & -2.901 \\
\hline 3757.759 & 73436 & (o) & 2.5 & 100040 & (e) & 1.5 & -3.77 & $7.83 \mathrm{E}+04$ & $5.29 \mathrm{E}-05$ & -3.535 \\
\hline 3764.434 & 78109 & (o) & 1.5 & 104666 & (e) & 0.5 & -1.64 & $1.06 \mathrm{E}+07$ & $1.38 \mathrm{E}-02$ & \\
\hline 3796.638 & 74319 & (o) & 2.5 & 100650 & (e) & 2.5 & -2.04 & $4.16 \mathrm{E}+06$ & $1.87 \mathrm{E}-03$ & -2.316 \\
\hline 3805.404 & 73407 & (o) & 0.5 & 99678 & (e) & 0.5 & -3.78 & $7.31 \mathrm{E}+04$ & $9.87 \mathrm{E}-05$ & -3.564 \\
\hline 3813.623 & 74436 & (o) & 2.5 & 100650 & (e) & 2.5 & -2.61 & $1.12 \mathrm{E}+06$ & $5.04 \mathrm{E}-04$ & \\
\hline 3835.436 & 74718 & (o) & 1.5 & 100783 & (e) & 0.5 & -3.91 & $5.55 \mathrm{E}+04$ & $7.77 \mathrm{E}-05$ & -3.745 \\
\hline 3855.012 & 74718 & (o) & 1.5 & 100650 & (e) & 2.5 & -2.30 & $2.23 \mathrm{E}+06$ & $1.00 \mathrm{E}-03$ & -2.393 \\
\hline 3865.517 & 74920 & (o) & 0.5 & 100783 & (e) & 0.5 & -3.88 & $5.62 \mathrm{E}+04$ & $7.87 \mathrm{E}-05$ & \\
\hline 3875.152 & 74985 & (o) & 1.5 & 100783 & (e) & 0.5 & -3.58 & $1.11 \mathrm{E}+05$ & $1.55 \mathrm{E}-04$ & -3.933 \\
\hline 3886.723 & 74319 & (o) & 2.5 & 100040 & (e) & 1.5 & -2.45 & $1.54 \mathrm{E}+06$ & $1.04 \mathrm{E}-03$ & -2.921 \\
\hline 3904.524 & 74436 & (o) & 2.5 & 100040 & (e) & 1.5 & -2.75 & $7.63 \mathrm{E}+05$ & $5.15 \mathrm{E}-04$ & \\
\hline 3947.922 & 74718 & (o) & 1.5 & 100040 & (e) & 1.5 & -3.05 & $3.78 \mathrm{E}+05$ & $2.55 \mathrm{E}-04$ & -3.028 \\
\hline 3969.287 & 74854 & (o) & 0.5 & 100040 & (e) & 1.5 & -2.43 & $1.56 \mathrm{E}+06$ & $1.05 \mathrm{E}-03$ & -4.419 \\
\hline 4005.231 & 74718 & (o) & 1.5 & 99678 & (e) & 0.5 & -2.44 & $1.52 \mathrm{E}+06$ & $2.05 \mathrm{E}-03$ & -2.403 \\
\hline 4027.222 & 74854 & (o) & 0.5 & 99678 & (e) & 0.5 & -3.10 & $3.23 \mathrm{E}+05$ & $4.36 \mathrm{E}-04$ & \\
\hline 4100.849 & 80288 & (o) & 1.5 & 104666 & (e) & 0.5 & -2.11 & $3.27 \mathrm{E}+06$ & $4.25 \mathrm{E}-03$ & \\
\hline 4204.110 & 77713 & (o) & 1.5 & 101493 & (e) & 1.5 & -1.92 & $4.45 \mathrm{E}+06$ & $3.12 \mathrm{E}-03$ & -2.046 \\
\hline 4215.460 & 77777 & (o) & 0.5 & 101493 & (e) & 1.5 & -2.11 & $2.78 \mathrm{E}+06$ & $1.95 \mathrm{E}-03$ & -2.044 \\
\hline 4243.716 & 77935 & (o) & 2.5 & 101493 & (e) & 1.5 & -2.60 & $9.36 \mathrm{E}+05$ & $6.55 \mathrm{E}-04$ & -2.51 \\
\hline 4275.343 & 78109 & (o) & 1.5 & 101493 & (e) & 1.5 & -1.68 & $7.43 \mathrm{E}+06$ & $5.20 \mathrm{E}-03$ & -1.56 \\
\hline 4288.755 & 81233 & (o) & 2.5 & 104543 & (e) & 3.5 & -1.62 & $9.46 \mathrm{E}+06$ & $2.72 \mathrm{E}-03$ & -1.526 \\
\hline 4333.501 & 77713 & (o) & 1.5 & 100783 & (e) & 0.5 & -2.26 & $1.91 \mathrm{E}+06$ & $2.67 \mathrm{E}-03$ & -2.183 \\
\hline 4345.561 & 77777 & (o) & 0.5 & 100783 & (e) & 0.5 & -1.90 & $4.24 \mathrm{E}+06$ & $5.94 \mathrm{E}-03$ & -1.851 \\
\hline 4409.225 & 78109 & (o) & 1.5 & 100783 & (e) & 0.5 & -2.32 & $1.59 \mathrm{E}+06$ & $2.23 \mathrm{E}-03$ & -2.218 \\
\hline 4477.646 & 77713 & (o) & 1.5 & 100040 & (e) & 1.5 & -3.63 & $7.52 \mathrm{E}+04$ & $5.08 \mathrm{E}-05$ & -3.74 \\
\hline 4490.523 & 77777 & (o) & 0.5 & 100040 & (e) & 1.5 & -3.67 & $6.79 \mathrm{E}+04$ & $4.58 \mathrm{E}-05$ & -3.583 \\
\hline 4551.509 & 77713 & (o) & 1.5 & 99678 & (e) & 0.5 & -3.83 & $4.64 \mathrm{E}+04$ & $6.26 \mathrm{E}-05$ & -3.614 \\
\hline 4558.539 & 78109 & (o) & 1.5 & 100040 & (e) & 1.5 & -3.43 & $1.17 \mathrm{E}+05$ & $7.90 \mathrm{E}-05$ & -3.246 \\
\hline 4564.815 & 77777 & (o) & 0.5 & 99678 & (e) & 0.5 & -3.77 & $5.22 \mathrm{E}+04$ & $7.05 \mathrm{E}-05$ & -3.932 \\
\hline 4635.118 & 78109 & (o) & 1.5 & 99678 & (e) & 0.5 & -3.72 & $5.72 \mathrm{E}+04$ & $7.72 \mathrm{E}-05$ & -3.418 \\
\hline 4714.594 & 80288 & (o) & 1.5 & 101493 & (e) & 1.5 & -2.00 & $3.20 \mathrm{E}+06$ & $2.24 \mathrm{E}-03$ & -2.041 \\
\hline 4744.159 & 80420 & (o) & 2.5 & 101493 & (e) & 1.5 & -1.33 & $1.49 \mathrm{E}+07$ & $1.04 \mathrm{E}-02$ & -1.351 \\
\hline 4872.316 & 63802 & (o) & 0.5 & 84320 & (e) & 1.5 & -3.18 & $1.89 \mathrm{E}+05$ & $1.37 \mathrm{E}-04$ & -3.042 \\
\hline 4877.924 & 80288 & (o) & 1.5 & 100783 & (e) & 0.5 & -1.65 & $6.76 \mathrm{E}+06$ & $9.46 \mathrm{E}-03$ & -1.651 \\
\hline 4892.456 & 64062 & (o) & 1.5 & 84496 & (e) & 2.5 & -3.23 & $1.67 \mathrm{E}+05$ & $8.07 \mathrm{E}-05$ & -2.947 \\
\hline 4898.663 & 63802 & (o) & 0.5 & 84210 & (e) & 0.5 & -2.92 & $3.34 \mathrm{E}+05$ & $4.84 \mathrm{E}-04$ & -2.954 \\
\hline 4934.471 & 81233 & (o) & 2.5 & 101493 & (e) & 1.5 & -3.33 & $1.39 \mathrm{E}+05$ & $9.73 \mathrm{E}-05$ & -2.614 \\
\hline 4934.834 & 64062 & (o) & 1.5 & 84320 & (e) & 1.5 & -2.69 & $5.65 \mathrm{E}+05$ & $4.10 \mathrm{E}-04$ & -2.777 \\
\hline 4961.864 & 64062 & (o) & 1.5 & 84210 & (e) & 0.5 & -3.08 & $2.26 \mathrm{E}+05$ & $3.28 \mathrm{E}-04$ & -3.016 \\
\hline 4986.920 & 64449 & (o) & 2.5 & 84496 & (e) & 2.5 & -2.45 & $9.70 \mathrm{E}+05$ & $4.69 \mathrm{E}-04$ & -2.51 \\
\hline 5014.032 & 84605 & (o) & 2.5 & 104543 & (e) & 3.5 & -2.60 & $7.06 \mathrm{E}+05$ & $2.03 \mathrm{E}-04$ & -2.143 \\
\hline 5030.958 & 64449 & (o) & 2.5 & 84320 & (e) & 1.5 & -3.08 & $2.23 \mathrm{E}+05$ & $1.62 \mathrm{E}-04$ & -2.93 \\
\hline 5061.329 & 80288 & (o) & 1.5 & 100040 & (e) & 1.5 & -3.55 & $7.96 \mathrm{E}+04$ & $5.37 \mathrm{E}-05$ & -3.512 \\
\hline 5095.417 & 80420 & (o) & 2.5 & 100040 & (e) & 1.5 & -2.92 & $3.29 \mathrm{E}+05$ & $2.22 \mathrm{E}-04$ & -2.932 \\
\hline 5108.138 & 64924 & (o) & 3.5 & 84496 & (e) & 2.5 & -3.43 & $9.73 \mathrm{E}+04$ & $4.70 \mathrm{E}-05$ & -3.101 \\
\hline 5155.906 & 80288 & (o) & 1.5 & 99678 & (e) & 0.5 & -3.29 & $1.39 \mathrm{E}+05$ & $1.88 \mathrm{E}-04$ & -3.317 \\
\hline 5182.374 & 65029 & (o) & 0.5 & 84320 & (e) & 1.5 & -3.12 & $1.88 \mathrm{E}+05$ & $1.36 \mathrm{E}-04$ & -3.176 \\
\hline 5196.392 & 65257 & (o) & 3.5 & 84496 & (e) & 2.5 & -3.14 & $1.77 \mathrm{E}+05$ & $8.56 \mathrm{E}-05$ & -3.231 \\
\hline 5212.191 & 65029 & (o) & 0.5 & 84210 & (e) & 0.5 & -3.15 & $1.76 \mathrm{E}+05$ & $2.55 \mathrm{E}-04$ & -3.248 \\
\hline 5216.761 & 65156 & (o) & 2.5 & 84320 & (e) & 1.5 & -3.67 & $5.17 \mathrm{E}+04$ & $3.75 \mathrm{E}-05$ & -3.784 \\
\hline 5239.683 & 85587 & (o) & 1.5 & 104666 & (e) & 0.5 & -3.72 & $4.62 \mathrm{E}+04$ & $6.01 \mathrm{E}-05$ & \\
\hline 5327.741 & 85779 & (o) & 2.5 & 104543 & (e) & 3.5 & -3.33 & $1.07 \mathrm{E}+05$ & $3.08 \mathrm{E}-05$ & -3.942 \\
\hline 5363.625 & 82854 & (o) & 0.5 & 101493 & (e) & 1.5 & -3.50 & $7.84 \mathrm{E}+04$ & $5.49 \mathrm{E}-05$ & -3.514 \\
\hline
\end{tabular}


Table 4 (continued)

\begin{tabular}{|c|c|c|c|c|c|c|c|c|c|c|}
\hline $\begin{array}{c}\text { Wavelength } \\
(\AA)\end{array}$ & $\begin{array}{c}E \text { (lower) } \\
\left(\mathrm{cm}^{-1}\right)\end{array}$ & Parity (lower) & $J$ (lower) & $\begin{array}{c}E \text { (upper) } \\
\left(\mathrm{cm}^{-1}\right)\end{array}$ & Parity (upper) & $J$ (upper) & $\log (g f)(\mathrm{HFR}+\mathrm{CPOL})$ & $\begin{array}{c}g A(\mathrm{HFR}+\mathrm{CPOL}) \\
\left(\mathrm{s}^{-1}\right)\end{array}$ & $B F(\mathrm{HFR}+\mathrm{CPOL})$ & $\begin{array}{c}\log (g f) \\
\text { (Kurucz) }\end{array}$ \\
\hline 5382.689 & 82920 & (o) & 1.5 & 101493 & (e) & 1.5 & -2.75 & $4.28 \mathrm{E}+05$ & $3.00 \mathrm{E}-04$ & -2.831 \\
\hline 5510.888 & 66355 & (o) & 1.5 & 84496 & (e) & 2.5 & -1.66 & $4.87 \mathrm{E}+06$ & $2.35 \mathrm{E}-03$ & -1.645 \\
\hline 5534.428 & 66256 & (o) & 0.5 & 84320 & (e) & 1.5 & -2.07 & $1.89 \mathrm{E}+06$ & $1.37 \mathrm{E}-03$ & -2.073 \\
\hline 5542.991 & 86507 & (o) & 2.5 & 104543 & (e) & 3.5 & -3.96 & $2.42 \mathrm{E}+04$ & $6.96 \mathrm{E}-06$ & -4.173 \\
\hline 5561.226 & 86566 & (o) & 2.5 & 104543 & (e) & 3.5 & -3.50 & $6.86 \mathrm{E}+04$ & $1.97 \mathrm{E}-05$ & -3.802 \\
\hline 5564.716 & 66355 & (o) & 1.5 & 84320 & (e) & 1.5 & -1.95 & $2.46 \mathrm{E}+06$ & $1.78 \mathrm{E}-03$ & -1.91 \\
\hline 5568.447 & 66256 & (o) & 0.5 & 84210 & (e) & 0.5 & -2.09 & $1.79 \mathrm{E}+06$ & $2.60 \mathrm{E}-03$ & -2.066 \\
\hline 5576.032 & 82854 & (o) & 0.5 & 100783 & (e) & 0.5 & -2.97 & $2.44 \mathrm{E}+05$ & $3.42 \mathrm{E}-04$ & -3.049 \\
\hline 5596.638 & 82920 & (o) & 1.5 & 100783 & (e) & 0.5 & -3.75 & $3.95 \mathrm{E}+04$ & $5.53 \mathrm{E}-05$ & \\
\hline 5599.109 & 66355 & (o) & 1.5 & 84210 & (e) & 0.5 & -2.84 & $3.13 \mathrm{E}+05$ & $4.54 \mathrm{E}-04$ & -2.765 \\
\hline 5601.872 & 66649 & (o) & 1.5 & 84496 & (e) & 2.5 & -2.87 & $2.84 \mathrm{E}+05$ & $1.37 \mathrm{E}-04$ & -4.449 \\
\hline 5626.270 & 66727 & (o) & 2.5 & 84496 & (e) & 2.5 & -1.99 & $2.21 \mathrm{E}+06$ & $1.07 \mathrm{E}-03$ & -1.958 \\
\hline 5633.086 & 86919 & (o) & 1.5 & 104666 & (e) & 0.5 & -3.79 & $3.44 \mathrm{E}+04$ & $4.47 \mathrm{E}-05$ & \\
\hline 5682.387 & 66727 & (o) & 2.5 & 84320 & (e) & 1.5 & -3.17 & $1.42 \mathrm{E}+05$ & $1.03 \mathrm{E}-04$ & -3.197 \\
\hline 5693.054 & 66649 & (o) & 1.5 & 84210 & (e) & 0.5 & -2.68 & $4.32 \mathrm{E}+05$ & $6.26 \mathrm{E}-04$ & -3.171 \\
\hline 5718.080 & 67012 & (o) & 2.5 & 84496 & (e) & 2.5 & -2.55 & $5.69 \mathrm{E}+05$ & $2.75 \mathrm{E}-04$ & -2.556 \\
\hline 5729.625 & 66872 & (o) & 0.5 & 84320 & (e) & 1.5 & -3.94 & $2.37 \mathrm{E}+04$ & $1.72 \mathrm{E}-05$ & -4.26 \\
\hline 5737.232 & 67070 & (o) & 1.5 & 84496 & (e) & 2.5 & -3.96 & $2.24 \mathrm{E}+04$ & $1.08 \mathrm{E}-05$ & -3.086 \\
\hline 5766.094 & 66872 & (o) & 0.5 & 84210 & (e) & 0.5 & -3.43 & $7.58 \mathrm{E}+04$ & $1.10 \mathrm{E}-04$ & -4.063 \\
\hline 5776.052 & 67012 & (o) & 2.5 & 84320 & (e) & 1.5 & -1.80 & $3.14 \mathrm{E}+06$ & $2.28 \mathrm{E}-03$ & -1.839 \\
\hline 5795.595 & 67070 & (o) & 1.5 & 84320 & (e) & 1.5 & -2.48 & $6.57 \mathrm{E}+05$ & $4.76 \mathrm{E}-04$ & -2.693 \\
\hline 5825.267 & 67334 & (o) & 3.5 & 84496 & (e) & 2.5 & -1.73 & $3.74 \mathrm{E}+06$ & $1.81 \mathrm{E}-03$ & -2.959 \\
\hline 5832.912 & 67070 & (o) & 1.5 & 84210 & (e) & 0.5 & -2.28 & $1.04 \mathrm{E}+06$ & $1.51 \mathrm{E}-03$ & -2.182 \\
\hline 5840.759 & 67379 & (o) & 1.5 & 84496 & (e) & 2.5 & -3.44 & $7.17 \mathrm{E}+04$ & $3.47 \mathrm{E}-05$ & -3.641 \\
\hline 5843.420 & 67387 & (o) & 2.5 & 84496 & (e) & 2.5 & -2.09 & $1.61 \mathrm{E}+06$ & $7.78 \mathrm{E}-04$ & -2.089 \\
\hline 5845.593 & 67393 & (o) & 3.5 & 84496 & (e) & 2.5 & -1.52 & $5.97 \mathrm{E}+06$ & $2.89 \mathrm{E}-03$ & -1.349 \\
\hline 5888.992 & 67344 & (o) & 2.5 & 84320 & (e) & 1.5 & -3.43 & $7.20 \mathrm{E}+04$ & $5.22 \mathrm{E}-05$ & -3.545 \\
\hline 5901.258 & 67379 & (o) & 1.5 & 84320 & (e) & 1.5 & -2.27 & $1.05 \mathrm{E}+06$ & $7.61 \mathrm{E}-04$ & -2.225 \\
\hline 5903.975 & 67387 & (o) & 2.5 & 84320 & (e) & 1.5 & -1.79 & $3.08 \mathrm{E}+06$ & $2.23 \mathrm{E}-03$ & -1.79 \\
\hline 5939.953 & 67379 & (o) & 1.5 & 84210 & (e) & 0.5 & -1.88 & $2.49 \mathrm{E}+06$ & $3.61 \mathrm{E}-03$ & -1.903 \\
\hline 6012.350 & 67868 & (o) & 2.5 & 84496 & (e) & 2.5 & -3.59 & $4.71 \mathrm{E}+04$ & $2.28 \mathrm{E}-05$ & -3.739 \\
\hline 6013.227 & 67870 & (o) & 1.5 & 84496 & (e) & 2.5 & -3.34 & $8.44 \mathrm{E}+04$ & $4.08 \mathrm{E}-05$ & -3.522 \\
\hline 6015.093 & 67875 & (o) & 3.5 & 84496 & (e) & 2.5 & -2.45 & $6.43 \mathrm{E}+05$ & $3.11 \mathrm{E}-04$ & -2.182 \\
\hline 6073.439 & 67860 & (o) & 0.5 & 84320 & (e) & 1.5 & -3.38 & $7.45 \mathrm{E}+04$ & $5.40 \mathrm{E}-05$ & -3.416 \\
\hline 6076.476 & 67868 & (o) & 2.5 & 84320 & (e) & 1.5 & -2.62 & $4.29 \mathrm{E}+05$ & $3.11 \mathrm{E}-04$ & -2.406 \\
\hline 6077.372 & 67870 & (o) & 1.5 & 84320 & (e) & 1.5 & -3.66 & $3.99 \mathrm{E}+04$ & $2.89 \mathrm{E}-05$ & -3.846 \\
\hline 6114.432 & 67860 & (o) & 0.5 & 84210 & (e) & 0.5 & -3.59 & $4.53 \mathrm{E}+04$ & $6.57 \mathrm{E}-05$ & -3.486 \\
\hline 6174.920 & 68306 & (o) & 1.5 & 84496 & (e) & 2.5 & -3.20 & $1.16 \mathrm{E}+05$ & $5.61 \mathrm{E}-05$ & -3.753 \\
\hline 6192.783 & 68583 & (o) & 2.5 & 84727 & (e) & 3.5 & -3.54 & $4.77 \mathrm{E}+04$ & $1.73 \mathrm{E}-05$ & -4.275 \\
\hline 6224.119 & 88604 & (o) & 1.5 & 104666 & (e) & 0.5 & -2.96 & $1.89 \mathrm{E}+05$ & $2.46 \mathrm{E}-04$ & \\
\hline 6242.581 & 68306 & (o) & 1.5 & 84320 & (e) & 1.5 & -3.95 & $2.00 \mathrm{E}+04$ & $1.45 \mathrm{E}-05$ & \\
\hline 6285.896 & 68306 & (o) & 1.5 & 84210 & (e) & 0.5 & -3.00 & $1.77 \mathrm{E}+05$ & $2.57 \mathrm{E}-04$ & -3.724 \\
\hline 6343.890 & 85486 & (o) & 0.5 & 101245 & (e) & 1.5 & -2.76 & $2.79 \mathrm{E}+05$ & $1.88 \mathrm{E}-04$ & -2.579 \\
\hline 6384.550 & 85587 & (o) & 1.5 & 101245 & (e) & 1.5 & -3.17 & $1.08 \mathrm{E}+05$ & $7.29 \mathrm{E}-05$ & -2.982 \\
\hline 6556.092 & 69478 & (o) & 2.5 & 84727 & (e) & 3.5 & -3.72 & $2.87 \mathrm{E}+04$ & $1.04 \mathrm{E}-05$ & -4.488 \\
\hline 6636.539 & 85587 & (o) & 1.5 & 100650 & (e) & 2.5 & -3.79 & $2.40 \mathrm{E}+04$ & $1.08 \mathrm{E}-05$ & -3.587 \\
\hline 6656.965 & 69478 & (o) & 2.5 & 84496 & (e) & 2.5 & -2.77 & $2.51 \mathrm{E}+05$ & $1.21 \mathrm{E}-04$ & -2.887 \\
\hline 6669.473 & 69506 & (o) & 3.5 & 84496 & (e) & 2.5 & -1.69 & $3.00 \mathrm{E}+06$ & $1.45 \mathrm{E}-03$ & -1.653 \\
\hline 6671.276 & 86507 & (o) & 2.5 & 101493 & (e) & 1.5 & -2.33 & $7.01 \mathrm{E}+05$ & $4.91 \mathrm{E}-04$ & -2.423 \\
\hline 6677.293 & 69348 & (o) & 1.5 & 84320 & (e) & 1.5 & -3.61 & $3.81 \mathrm{E}+04$ & $2.76 \mathrm{E}-05$ & -3.71 \\
\hline 6722.277 & 85779 & (o) & 2.5 & 100650 & (e) & 2.5 & -2.83 & $2.13 \mathrm{E}+05$ & $9.59 \mathrm{E}-05$ & -2.625 \\
\hline 6726.875 & 69348 & (o) & 1.5 & 84210 & (e) & 0.5 & -3.52 & $4.56 \mathrm{E}+04$ & $6.61 \mathrm{E}-05$ & -3.45 \\
\hline 6728.966 & 69639 & (o) & 1.5 & 84496 & (e) & 2.5 & -3.49 & $4.75 \mathrm{E}+04$ & $2.30 \mathrm{E}-05$ & -3.485 \\
\hline 6735.669 & 69478 & (o) & 2.5 & 84320 & (e) & 1.5 & -2.54 & $4.18 \mathrm{E}+05$ & $3.03 \mathrm{E}-04$ & -2.552 \\
\hline 6809.392 & 69639 & (o) & 1.5 & 84320 & (e) & 1.5 & -2.42 & $5.31 \mathrm{E}+05$ & $3.85 \mathrm{E}-04$ & -2.393 \\
\hline 6810.785 & 86566 & (o) & 2.5 & 101245 & (e) & 1.5 & -3.66 & $3.22 \mathrm{E}+04$ & $2.17 \mathrm{E}-05$ & -2.955 \\
\hline 6851.119 & 69903 & (o) & 3.5 & 84496 & (e) & 2.5 & -3.36 & $6.19 \mathrm{E}+04$ & $2.99 \mathrm{E}-05$ & -3.595 \\
\hline 6859.728 & 86919 & (o) & 1.5 & 101493 & (e) & 1.5 & -3.16 & $9.96 \mathrm{E}+04$ & $6.97 \mathrm{E}-05$ & -3.331 \\
\hline 6860.753 & 86079 & (o) & 3.5 & 100650 & (e) & 2.5 & -2.18 & $9.04 \mathrm{E}+05$ & $4.07 \mathrm{E}-04$ & -1.977 \\
\hline 6860.963 & 69639 & (o) & 1.5 & 84210 & (e) & 0.5 & -2.03 & $1.29 \mathrm{E}+06$ & $1.87 \mathrm{E}-03$ & -1.998 \\
\hline 6869.064 & 85486 & (o) & 0.5 & 100040 & (e) & 1.5 & -3.56 & $3.77 E+04$ & $2.54 \mathrm{E}-05$ & -3.365 \\
\hline 6874.944 & 69954 & (o) & 2.5 & 84496 & (e) & 2.5 & -2.47 & $4.94 \mathrm{E}+05$ & $2.39 \mathrm{E}-04$ & -2.434 \\
\hline 6916.760 & 85587 & (o) & 1.5 & 100040 & (e) & 1.5 & -2.75 & $2.42 \mathrm{E}+05$ & $1.63 \mathrm{E}-04$ & -2.55 \\
\hline 6940.497 & 90262 & (o) & 1.5 & 104666 & (e) & 0.5 & -3.91 & $1.74 \mathrm{E}+04$ & $2.26 \mathrm{E}-05$ & \\
\hline 6958.919 & 69954 & (o) & 2.5 & 84320 & (e) & 1.5 & -1.94 & $1.61 \mathrm{E}+06$ & $1.17 \mathrm{E}-03$ & -1.923 \\
\hline 7009.942 & 85779 & (o) & 2.5 & 100040 & (e) & 1.5 & -2.44 & $4.76 \mathrm{E}+05$ & $3.21 \mathrm{E}-04$ & -2.246 \\
\hline
\end{tabular}


Table 4 (continued)

\begin{tabular}{|c|c|c|c|c|c|c|c|c|c|c|}
\hline $\begin{array}{c}\text { Wavelength } \\
(\AA)\end{array}$ & $\begin{array}{c}E \text { (lower) } \\
\left(\mathrm{cm}^{-1}\right)\end{array}$ & Parity (lower) & $J$ (lower) & $\begin{array}{c}E \text { (upper) } \\
\left(\mathrm{cm}^{-1}\right)\end{array}$ & Parity (upper) & $J$ (upper) & $\log (g f)(\mathrm{HFR}+\mathrm{CPOL})$ & $\begin{array}{c}g A(\mathrm{HFR}+\mathrm{CPOL}) \\
\left(\mathrm{s}^{-1}\right)\end{array}$ & $B F(\mathrm{HFR}+\mathrm{CPOL})$ & $\begin{array}{c}\log (g f) \\
\text { (Kurucz) }\end{array}$ \\
\hline 7044.435 & 85486 & (o) & 0.5 & 99678 & (e) & 0.5 & -2.85 & $1.85 \mathrm{E}+05$ & $2.50 \mathrm{E}-04$ & -2.653 \\
\hline 7094.607 & 85587 & (o) & 1.5 & 99678 & (e) & 0.5 & -2.83 & $1.90 \mathrm{E}+05$ & $2.57 \mathrm{E}-04$ & -2.633 \\
\hline 7106.057 & 70427 & (o) & 3.5 & 84496 & (e) & 2.5 & -3.12 & $9.93 \mathrm{E}+04$ & $4.80 \mathrm{E}-05$ & -2.903 \\
\hline 7139.197 & 70317 & (o) & 2.5 & 84320 & (e) & 1.5 & -3.78 & $2.16 \mathrm{E}+04$ & $1.57 \mathrm{E}-05$ & -3.186 \\
\hline 7211.034 & 86919 & (o) & 1.5 & 100783 & (e) & 0.5 & -2.59 & $3.34 \mathrm{E}+05$ & $4.68 \mathrm{E}-04$ & -2.707 \\
\hline 7278.269 & 70584 & (o) & 2.5 & 84320 & (e) & 1.5 & -3.40 & $5.09 \mathrm{E}+04$ & $3.69 \mathrm{E}-05$ & -3.387 \\
\hline 7327.505 & 70852 & (o) & 3.5 & 84496 & (e) & 2.5 & -3.49 & $4.07 \mathrm{E}+04$ & $1.97 \mathrm{E}-05$ & -3.354 \\
\hline 7387.392 & 86507 & (o) & 2.5 & 100040 & (e) & 1.5 & -3.73 & $2.29 \mathrm{E}+04$ & $1.55 \mathrm{E}-05$ & -3.653 \\
\hline 7404.052 & 86566 & (o) & 2.5 & 100069 & (e) & 3.5 & -2.27 & $6.48 \mathrm{E}+05$ & $2.27 \mathrm{E}-04$ & -2.165 \\
\hline 7756.678 & 88604 & (o) & 1.5 & 101493 & (e) & 1.5 & -3.33 & $5.17 \mathrm{E}+04$ & $3.62 \mathrm{E}-05$ & -2.969 \\
\hline 9345.279 & 93969 & (o) & 0.5 & 104666 & (e) & 0.5 & -3.84 & $1.19 \mathrm{E}+04$ & $1.55 \mathrm{E}-05$ & \\
\hline 9603.253 & 94256 & (o) & 1.5 & 104666 & (e) & 0.5 & -3.32 & $3.51 \mathrm{E}+04$ & $4.56 \mathrm{E}-05$ & \\
\hline 11871.802 & 96245 & (o) & 0.5 & 104666 & (e) & 0.5 & -2.30 & $2.23 \mathrm{E}+05$ & $2.90 \mathrm{E}-04$ & \\
\hline
\end{tabular}

